\title{
ON THE LIMIT AS THE DENSITY RATIO TENDS TO ZERO FOR TWO PERFECT INCOMPRESSIBLE FLUIDS SEPARATED BY A SURFACE OF DISCONTINUITY
}

\author{
C.H. ARTHUR CHENG, DANIEL COUTAND, AND STEVE SHKOLLER
}

\begin{abstract}
We study the asymptotic limit as the density ratio $\rho^{-} / \rho^{+} \rightarrow 0$, where $\rho^{+}$and $\rho^{-}$are the densities of two perfect incompressible 2-D/3-D fluids, separated by a surface of discontinuity along which the pressure jump is proportional to the mean curvature of the moving surface. Mathematically, the fluid motion is governed by the two-phase incompressible Euler equations with vortex sheet data. By rescaling, we assume the density $\rho^{+}$of the inner fluid is fixed, while the density $\rho^{-}$of the outer fluid is set to $\epsilon$. We prove that solutions of the free-boundary Euler equations in vacuum are obtained in the limit as $\epsilon \rightarrow 0$.
\end{abstract}

\section{INTRODUCTION}

1.1. The water wave problem. A number of articles have recently appeared that focus on the analysis of the one-phase free-boundary incompressible Euler equations, in either irrotational form or with vorticy, in both 2-D and 3-D, and with or without surface tension effects on the free surface. See $[6,8,10,11,12$, $13,14,18,19,21]$ and the references therein. In irrotational form, the one-phase incompressible Euler equations with free-surface are often referred to as the water wave equations for the motion of the interface, since irrotationality decouples the motion of the liquid from that of the free-surface wave motion. The water wave equations typically model the motion of a liquid drop inside of air, or the waves on the surface of the ocean underneath the atmosphere (of course, air can be replaced with any other incompressible liquid with very small relative density). In particular, suppose that the density of the liquid is denoted by $\rho^{+}$while the density of air (or the lighter liquid) by $\rho^{-}$. Even when $\rho^{-} \ll \rho^{+}$, the motion of the liquid-air system is more accurately modeled by the two-phase Euler equations, which in irrotational form lead to the equation of motion for vortex sheets.

The jump discontinuity in the tangential component of velocity across the material interface, which appears in the two-phase Euler model, is responsible for the ill-posedness of this system of PDE when surface tension effects are ignored (see [9] and [20]). On the other hand, in the presence of surface tension, the two-phase system is well-posed. See [4] for existence and uniqueness of solutions to two-phase (rotational) Euler equations, and see $[1,2,3]$ for the proof of well-posedness for the irrotational problem. Also, see [15] for an infinite-dimensional geometric approach to a priori estimates of the general problem. With surface tension included, the pressure experiences a jump discontinuity proportional to the mean curvature of the vortex sheet as we describe below. The two-phase system is a great deal more difficult to simulate computationally or study analytically, so it is of significant interest to rigorously establish the convergence of solutions to the two-phase equations (vortex sheets) to those of the one-phase model (water waves) in the limit as 
$\rho^{-} / \rho^{+} \rightarrow 0$. The purpose of this paper is to prove this asymptotic result, without any irrotationality assumptions on the fluids. We state our results for the case that the space dimension is either 2 or 3 , but we note that with additional regularity assumptions on the data, our results are valid for any space dimension great than 1.

1.2. The two-phase Euler equations in Eulerian variables. For $\mathfrak{n}=2$ or 3 , let $\mathcal{D} \subseteq \mathbb{R}^{\mathfrak{n}}$ denote an open, bounded set, which comprises the volume occupied by two incompressible and inviscid fluids with different densities. At the initial time $t=0$, we let $\Omega^{+}$denote the volume occupied by the inner fluid with density $\rho^{+}$and we let $\Omega^{-}$denote the volume occupied by the outer fluid with density $\rho^{-}$. Mathematically, the sets $\Omega^{+}$and $\Omega^{-}$denote two disjoint open bounded subsets of $\mathcal{D}$ such that $\overline{\mathcal{D}}=\overline{\Omega^{+}} \cup \overline{\Omega^{-}}$and $\Omega^{+} \cap \Omega^{-}=\emptyset$. The material interface at time $t=0$ is given by $\Gamma:=\overline{\Omega^{+}} \cap \overline{\Omega^{-}}$, and $\partial \mathcal{D}=\partial \Omega^{-}-\Gamma$.

Let $\Omega^{+}(t)$ and $\Omega^{-}(t)$ denote the time-dependent volumes of the inner and outer fluids, respectively, separated by the moving material interface $\Gamma(t)$. Let $u^{ \pm}$and $p^{ \pm}$denote the velocity field and pressure function, respectively, in $\Omega^{ \pm}(t)$. Then the so-called vortex sheet problem, given by the solution of the incompressible Euler equations for the motion of two fluids separated by a moving surface of discontinuity, can be written as

$$
\begin{aligned}
\rho^{ \pm}\left(u_{t}^{ \pm}+u^{ \pm} \cdot D u^{ \pm}\right)+D p^{ \pm} & =0 & & \text { in } \quad \Omega^{ \pm}(t), \\
\operatorname{div} u^{ \pm} & =0 & & \text { in } \quad \Omega^{ \pm}(t), \\
{[p]_{ \pm} } & =\sigma H & & \text { on } \quad \Gamma(t), \\
{[u \cdot n]_{ \pm} } & =0 & & \text { on } \quad \Gamma(t), \\
u^{-} \cdot n & =0 & & \text { on } \quad \partial \mathcal{D}, \\
u(0) & =u_{0} & & \text { on } \quad\{t=0\} \times \mathcal{D}, \\
\mathcal{V}(\Gamma(t)) & =u^{+}(t) \cdot n(t), & &
\end{aligned}
$$

where $\mathcal{V}(\Gamma(t))$ denotes the speed of the moving interface $\Gamma(t)$ in the normal direction, and $n(t)$ denotes the outward-pointing unit normal to $\partial \Omega^{+}(t)$; thus, (1.1g) indicates that the vortex sheet $\Gamma(t)$ moves with the normal component of the fluid velocity. $\rho^{+}$and $\rho^{-}$are the densities of the two fluids occupying $\Omega^{+}(t)$ and $\Omega^{-}(t)$, respectively, $H(t)$ is twice the mean curvature of $\Gamma(t)$, and $\sigma>0$ is the surface tension parameter which we will henceforth set to one.

In [4], we proved the existence and uniqueness of the solutions to (1.1). See also $[1,2,3]$ for the proof of well-posedness for the irrotational problem, and [15] for an infinite-dimensional geometric approach to a priori estimates of the general problem.

By rescaling, if necessary, we may assume that

$$
\rho^{+}=1 \text { and that } \rho^{-}=\epsilon \ll 1 \text {. }
$$

Letting $u_{\epsilon}^{ \pm}$denote the solutions of (1.1), the main objective of this article is to to study the asymptotic behavior of the solutions $u_{\epsilon}^{ \pm}$as $\epsilon \rightarrow 0$.

\subsection{Notation.}

1.3.1. Sobolev norms on $\Omega^{ \pm}$and $\Gamma$. Let $H^{s}\left(\Omega^{+}\right)$denote $H^{s}\left(\Omega^{+} ; \mathbb{R}\right)$ for scalar functions or $H^{s}\left(\Omega^{+} ; \mathbb{R}^{\mathfrak{n}}\right)$ for vector fields, and let $H^{s}\left(\Omega^{-}\right)$denote $H^{s}\left(\Omega^{-} ; \mathbb{R}\right) / \mathbb{R}$ for a 
scalar functions with zero average or $H^{s}\left(\Omega^{-} ; \mathbb{R}^{\mathfrak{n}}\right)$ for vector fields. We denote the $H^{s}\left(\Omega^{ \pm}\right)$-norms by

$$
\left\|w^{+}\right\|_{s,+}=\left\|w^{+}\right\|_{H^{s}\left(\Omega^{+}\right)} \quad \text { and } \quad\left\|w^{-}\right\|_{s,-}=\left\|w^{-}\right\|_{H^{s}\left(\Omega^{-}\right)} .
$$

The $H^{s}(\Gamma)$ - and $H^{s}(\partial \mathcal{D})$-norms are denoted by

$$
\left|w^{+}\right|_{s}=\left\|w^{+}\right\|_{H^{s}(\Gamma)},\left|w^{-}\right|_{s}=\left\|w^{-}\right\|_{H^{s}(\Gamma)}, \text { and }|w|_{s, \partial \mathcal{D}}=\|w\|_{H^{s}(\partial \mathcal{D})} .
$$

For simplicity, we also use $\|w\|_{s, \pm}^{2}$ and $|w|_{s, \pm}^{2}$ to denote $\left\|w^{+}\right\|_{s,+}^{2}+\left\|w^{-}\right\|_{s,-}^{2}$ and $\left|w^{+}\right|_{s}^{2}+\left|w^{-}\right|_{s}^{2}$, respectively, that is,

$$
\begin{aligned}
\|w\|_{s, \pm}^{2} & =\left\|w^{+}\right\|_{s,+}^{2}+\left\|w^{-}\right\|_{s,-}^{2}, \\
|w|_{s, \pm}^{2} & =\left|w^{+}\right|_{s}^{2}+\left|w^{-}\right|_{s}^{2} .
\end{aligned}
$$

We also use $\langle\cdot, \cdot\rangle_{H^{1}\left(\Omega^{+}\right)},\langle\cdot, \cdot\rangle_{H^{1}\left(\Omega^{-}\right)}$and $\langle\cdot, \cdot\rangle_{H^{0.5}(\Gamma)}$ to denote the duality pairing between $H^{1}\left(\Omega^{+}\right)$and $H^{1}\left(\Omega^{+}\right)^{\prime}$, the duality pairing between $H^{1}\left(\Omega^{-}\right)$and $H^{1}\left(\Omega^{-}\right)^{\prime}$, and the duality paring between $H^{0.5}(\Gamma)$ and $H^{-0.5}(\Gamma)$, respectively.

1.3.2. Einstein summation convention. Repeated Latin indices are summed from 1 to $\mathfrak{n}$, while repeated Greek indices are summed from 1 to $\mathfrak{n}-1$. For example,

$$
f^{\alpha} g_{\alpha}:=\sum_{\alpha=1}^{\mathfrak{n}-1} f^{\alpha} g_{\alpha} \quad \text { and } \quad f^{i} g_{i}:=\sum_{i=1}^{\mathfrak{n}} f^{i} g_{i} .
$$

1.3.3. The tangential derivative. Let $\left\{U_{\ell}\right\}_{\ell=1}^{K}$ denote an open covering of $\Gamma$, such that for each $\ell \in\{1,2, \cdots, K\}$, with

$$
\begin{aligned}
& V_{\ell}=B\left(0, r_{\ell}\right), \text { denoting the open ball of radius } r_{\ell} \text { centered at the origin and, } \\
& V_{\ell}^{+}=V_{\ell} \cap\left\{x_{\mathfrak{n}}>0\right\}, \\
& V_{\ell}^{-}=V_{\ell} \cap\left\{x_{\mathfrak{n}}<0\right\},
\end{aligned}
$$

there exist for $s \geq 3, H^{s}$-class charts $\theta_{\ell}$ which satisfy

$$
\begin{gathered}
\theta_{\ell}: V_{\ell} \rightarrow U_{\ell} \text { is an } H^{s} \text { diffeomorphism, } \\
\theta_{\ell}\left(V_{\ell}^{+}\right)=U_{\ell} \cap \Omega^{+}, \\
\theta_{\ell}\left(V_{\ell} \cap\left\{x_{\mathfrak{n}}=0\right\}\right)=U_{\ell} \cap \Gamma .
\end{gathered}
$$

Next, for $L>K$, let $\left\{U_{\ell}\right\}_{\ell=K+1}^{L}$ denote a family of open balls of radius $r_{\ell}$ contained in $\Omega$ such that $\left\{U_{\ell}\right\}_{\ell=1}^{L}$ is an open cover of $\Omega$, and let

$\left\{\xi_{\ell}\right\}_{\ell=1}^{L}$ denote a $\mathcal{C}^{\infty}$ partition of unity subordinate to this covering of $\Omega$.

We use $\bar{\partial}$ to denote the tangential derivative in $U_{\ell} \cap \Omega$. For a differentiable function $f$ on $\Omega$, the $\alpha$-th component of the tangential derivative of $f$ is given by

$$
f_{, \alpha}=\bar{\partial}_{\alpha} f=\frac{\partial}{\partial x_{\alpha}}\left[f \circ \theta_{\ell}\right] \circ \theta_{\ell}^{-1}=\left[\left(D f \circ \theta_{\ell}\right) \frac{\partial \theta_{\ell}}{\partial x_{\alpha}}\right] \circ \theta_{\ell}^{-1} .
$$

We use $f_{, i}$ to denote the i-th component of $D f$, where $D f$ is the gradient of $f$, or

$$
f_{, i}=\frac{\partial f}{\partial x_{i}} .
$$


1.3.4. The identity map e. The identity map on $\mathbb{R}^{n}$ is denoted by $e$ so that $e(x)=x$. For $\alpha=1,2$, we use the notation $e_{, \alpha}$ to denote the two tangent vectors to the reference material interface $\Gamma$; more specifically, in any local coordinate chart $V_{\ell}$, $e, \alpha$ denotes the tangent vectors $\frac{\partial \theta_{\ell}}{\partial x_{\alpha}}$. Note that

$$
\left[(D f) \circ \theta_{\ell}\right] \cdot e_{, \alpha}=f_{, \alpha} \circ \theta_{\ell} \quad \text { or } \quad\left(f_{, j} \circ \theta_{\ell}\right) e_{, \alpha}^{j}=f_{, \alpha} \circ \theta_{\ell} \text {. }
$$

1.3.5. $H^{s}$ norm of $\Gamma$. We defined the $H^{k}$-norm of $\Gamma$ to be

$$
|\Gamma|_{k}^{2}:=\sum_{\ell=1}^{K} \int_{\mathbb{R}}^{\mathfrak{n}-1} \xi_{\ell}\left|\partial_{\alpha_{1} \cdots \alpha_{k}}^{k} \theta_{\ell}\right|^{2} d x_{1} \cdots d x_{\mathfrak{n}-1} .
$$

The $H^{s}$-norm for any real $s \geq 0$ is defined by interpolation. We say that $\Gamma$ is of class $H^{s}$ (or $\left.\Gamma \in H^{s}\right)$ whenever $|\Gamma|_{s}<\infty$. The $H^{s}$-norm of $\partial \mathcal{D}$ is defined similarly.

1.3.6. Inner products and contractions. Given two vector $v$ and $w$ in $\mathbb{R}^{\mathfrak{n}}$, the inner product of $v$ and $w$ is denoted by $v \cdot w$, which in component is defined as

$$
v \cdot w=v^{i} w_{i}=\sum_{i=1}^{\mathfrak{n}} v^{i} w_{i}
$$

For two matrices $A$ and $B$, the contraction between $A$ and $B$, denoted by $A: B$, is the trace of the product of $A$ and $B$, which in component is defined as

$$
A: B=\operatorname{Tr}(A B)=A_{i}^{j} B_{j}^{i}=\sum_{i, j=1}^{\mathfrak{n}} A_{j}^{i} B_{i}^{j} .
$$

1.3.7. The transpose of matrices. Given any matrix $\mathcal{A}$, we use $\mathcal{A}^{T}$ to denote its transpose.

1.4. The arbitrary Lagrangian-Eulerian (ALE) formulation. Let $\eta^{+}$denote the Lagrangian flow map of $u^{+}$in $\Omega^{+}$, that is,

$$
\begin{aligned}
& \eta_{t}^{+}(x, t)=u^{+}\left(\eta^{+}(x, t), t\right) \quad \forall x \in \Omega^{+}, t>0, \\
& \eta^{+}(x, 0)=x \quad \forall x \in \Omega^{+} .
\end{aligned}
$$

By a theorem of Dacorogna and Moser [7], we can choose a volume preserving diffeomorphism $\bar{\psi}$ on $\Omega^{-}$such that

$$
\begin{aligned}
\operatorname{det}(D \bar{\psi}) & =1 & \forall x \in \Omega^{-}, \\
\bar{\psi} & =\eta^{+} & \forall x \in \Gamma, \\
\bar{\psi} & =e & \forall x \in \partial \mathcal{D} .
\end{aligned}
$$

Furthermore, the following elliptic estimate holds:

$$
\|\bar{\psi}\|_{4.5,-} \leq C\left[\left|\eta^{+}\right|_{4}+|\partial \mathcal{D}|_{4}\right] .
$$

We then define

$$
\psi(x, t)= \begin{cases}\eta^{+}(x, t) & x \in \overline{\Omega^{+}} \\ \bar{\psi}(x, t) & x \in \Omega^{-}\end{cases}
$$

REMARK 1. We emphasize that $\psi_{t}$ does not equal $v^{-}$in $\Omega^{-}$; on the other hand,

$$
\psi_{t}=v^{+} \text {on } \bar{\Omega}^{+} \text {. }
$$


Set $v=u \circ \psi, q=p \circ \psi$, and let $A=(D \psi)^{-1}$. Using the ALE variables, equations (1.1) are written as

$$
\begin{aligned}
v_{t}^{+i}+A_{i}^{k} q_{, k}^{+} & =0 & & \text { in } \quad(0, T) \times \Omega^{+}, \\
\epsilon v_{t}^{-i}+\epsilon \mathrm{W}^{j} v_{, j}^{-i}+A_{i}^{k} q_{, k}^{-} & =0 & & \text { in } \quad(0, T) \times \Omega^{-}, \\
A_{i}^{j} v_{, j}^{ \pm i} & =0 & & \text { in } \quad(0, T) \times \Omega^{ \pm}, \\
q^{+}-q^{-} & =-\Delta_{g} \eta \cdot n & & \text { on } \quad(0, T) \times \Gamma, \\
v^{+} \cdot n & =v^{-} \cdot n & & \text { on } \quad(0, T) \times \Gamma, \\
v^{-} \cdot N & =0 & & \text { on } \quad \partial \mathcal{D}, \\
\left(\psi(t), v(t), \Omega^{ \pm}(t)\right)_{t=0} & =\left(e, u_{0}, \Omega^{ \pm}\right), & &
\end{aligned}
$$

where $e(x)=x$ denotes the identity map on $\mathcal{D}, \mathrm{w}=A\left(v^{-}-\psi_{t}\right)$, and $n(t):=n(\psi(t))$ denotes the outward-point unit normal to $\partial \Omega^{+}(t)$ and evaluated at the point $\psi(t)$. With $N$ denoting the outward-point unit normal to $\partial \Omega^{+}$at $t=0$, we have the identity

$$
n(\psi(t))=\frac{A^{T} N}{\left|A^{T} N\right|}
$$

1.5. The higher-order energy function. With $\mathcal{S}(t)$ denoting the surface area of the vortex sheet $\Gamma(t)$, the physical energy function is given by $\left\|u^{+}\right\|_{L^{2}\left(\Omega^{+}(t)\right)}^{2}+$ $\epsilon\left\|u^{-}\right\|_{L^{2}\left(\Omega^{-}(t)\right)}^{2}+2 \sigma \mathcal{S}(t)$. While the physical energy is exactly conserved, it is much too weak to provide the necessary a priori control to pass to the limit as $\epsilon \rightarrow 0$. As such, we define the higher-order energy function $\mathcal{E}$

$$
\mathcal{E}(t)=\left|\bar{\partial} \eta^{+} \cdot N\right|_{3,+}^{2}+\|v\|_{3, \pm}^{2}+\left\|v_{t}\right\|_{1.5, \pm}^{2}+\left\|v_{t t}^{+}\right\|_{0,+}^{2}+\epsilon\left\|v_{t t}^{-}\right\|_{0,-}^{2} .
$$

Note that only $\epsilon\left\|v_{t t}^{-}\right\|_{0,-}^{2}$ has the asymptotic scaling parameter $\epsilon$.

1.6. The regularity of the solution to (1.1). With $\epsilon=\rho^{-} / \rho^{+}$, the following theorem is the main result in [4].

THEOREM (Well-posedness of (1.1)) Suppose that $\sigma>0$, and that $\Gamma:=\Gamma(0)$ is of class $H^{4}, \partial \mathcal{D}$ is of class $H^{3}$, and $u_{0}^{ \pm} \in H^{3}\left(\Omega^{ \pm}\right)$. Then, for all $\epsilon>0$, there exists $T_{\epsilon}>0$, and a solution $\left(u^{ \pm}(t), p^{ \pm}(t), \Omega^{ \pm}(t)\right)$ of (1.1) with $u^{ \pm} \in L^{\infty}\left(0, T ; H^{3}\left(\Omega^{ \pm}(t)\right)\right.$, $p^{ \pm} \in L^{\infty}\left(0, T ; H^{2.5}\left(\Omega^{ \pm}(t)\right)\right.$, and $\Gamma(t) \in H^{4}$. The solution is unique if $u_{0}^{ \pm} \in$ $H^{4.5}\left(\Omega^{ \pm}\right)$and $\Gamma \in H^{5.5}$.

Note that the time of existence $T_{\epsilon}$ depends crucially upon $\epsilon$, and that apriori $T_{\epsilon}$ may approach zero as $\epsilon \rightarrow 0$.

1.7. Main Result. Let $\Omega(t)=\Omega^{+}(t)$, and let $U$ denote the solution of the onephase free-surface incompressible Euler equations in vacuum, satisfying

$$
\begin{aligned}
U_{t}+U \cdot D U+D P & =0 & & \text { in } \quad \Omega(t), \\
\operatorname{div} U & =0 & & \text { in } \Omega(t), \\
P & =H & & \text { on } \Gamma(t), \\
U \cdot n & =0 & & \text { on } \Gamma(t), \\
u(0) & =u_{0} & & \text { on } \quad\{t=0\} \times \Omega, \\
\mathcal{V}(\Gamma(t)) & =U(t) \cdot n(t) . & &
\end{aligned}
$$


Theorem 1.1 (Main Theorem). Let $\epsilon=\rho^{-} / \rho^{+}$, and for $\epsilon>0$, let $u_{\epsilon}^{+}$denote the sequence of the solution to (1.1) in the inner phase $\Omega^{+}(t)$. Suppose that $u_{0}^{+}=$ $\left.u_{0}\right|_{\Omega^{+}} \in H^{3}\left(\Omega^{+}\right)$and $u_{0}^{-}=\left.u_{0}\right|_{\Omega^{-}} \in H^{3}\left(\Omega^{-}\right)$satisfying $u_{0}^{+} \cdot N=u_{0}^{-} \cdot N$ on $\Gamma$, where $\Gamma$ is of class $H^{4.5}$ and $\partial \mathcal{D}$ is smooth. Then there exists $T>0$, independent of $\epsilon$, such that the solution $u_{\epsilon}^{+}$o $\eta_{\epsilon}$ to (1.1) converges weakly to $U$ o $\eta$ in $L^{2}\left(0, T ; H^{3}\left(\Omega^{+}\right)\right)$ as $\epsilon \rightarrow 0$, where $\eta_{\epsilon}$ and $\eta$ are flows of $u_{\epsilon}^{+}$and $U$, respectively.

REMARK 2. Note that $u_{\epsilon}$ a priori only exists on the $\epsilon$-dependent time interval $\left(0, T_{\epsilon}\right)$; however, the main theorem shows that $T_{\epsilon}$ is in fact independent of $\epsilon$, and that $u_{\epsilon}$ exists on an $\epsilon$-independent interval $(0, T)$ for all $\epsilon>0$.

1.8. The structure of the proof and outline of the paper. The proof of Theorem 1.1 consists of several steps that we describe as follows. In Section 2, we review some well-known inequalities that we use throughout our analysis. In Section 3 , we establish estimates for the time derivatives of velocity and pressure, evaluated at time $t=0$. Section 4 is devoted to the derivation of $\epsilon$-independent estimates for our two-phase system. The fundamental difficulty resides in the estimates for the normal and tangential components of $v_{t t}^{-}$, which are founded on improved elliptic estimates (with respect to our estimates in [4]) for the pressure functions. Finally, in Section 5, we pass to the limit as $\epsilon \rightarrow 0$ and establish our main result.

\section{Preliminary Results}

2.1. The trace of the normal component of a vector field. A vector $u \in$ $L^{2}(\mathcal{O})$ with $\operatorname{div} u \in H^{1}(\mathcal{O})^{\prime}$ has a normal trace $u \cdot N \in H^{-0.5}(\partial \mathcal{O})$, where $N$ is the unit normal to the surface $\partial \mathcal{O}$, with the estimate

$$
\|u \cdot N\|_{H^{-0.5}(\partial \mathcal{O})}^{2} \leq C\left[\|u\|_{L^{2}(\mathcal{O})}^{2}+\|\operatorname{div} u\|_{H^{1}(\mathcal{O})^{\prime}}^{2}\right]
$$

where $C$ depends on $|\Gamma|_{s}$ for all $s>\frac{\mathfrak{n}+2}{2}$ (see, for example, [17]).

By the Piola identity, $A_{i}^{k}{ }_{k}=0$ (since $\operatorname{det} D \psi=1$ ), and the identity $A^{T} N=\sqrt{g} n$, letting $u^{j}=A_{i}^{j} w^{i}$ in (2.1) yields the Lagrangian normal trace estimate

$$
\|w \cdot n\|_{H^{-0.5}(\partial \mathcal{O})}^{2} \leq C\left[\|w\|_{L^{2}(\mathcal{O})}^{2}+\left\|A_{i}^{j} w_{, j}^{i}\right\|_{H^{1}(\mathcal{O})^{\prime}}^{2}\right],
$$

where $\mathcal{O}$ is either $\Omega^{+}$or $\Omega^{-}$.

\subsection{The Hodge decomposition elliptic estimate.}

Proposition 2.1. For $r \geq 2.5$, let $\mathcal{O}$ be $H^{r}$ domain, that is, $\partial \mathcal{O}$ is of class $H^{r-0.5}$. If $w \in L^{2}\left(\mathcal{O} ; \mathbb{R}^{3}\right)$ with $\operatorname{curl} w \in H^{r-1}(\mathcal{O})$, $\operatorname{div} w \in H^{r-1}(\mathcal{O})$, and $\bar{\partial} w \cdot N \in$ $H^{r-1.5}(\partial \mathcal{O})$, then there exists a constant $C$ depending on $|\partial \mathcal{O}|_{r-0.5}$ such that

$$
\begin{aligned}
\|w\|_{H^{r}(\mathcal{O})} \leq C\left(|\partial \mathcal{O}|_{r-0.5}\right) & {\left[\|w\|_{L^{2}(\mathcal{O})}+\|\operatorname{curl} w\|_{H^{r-1}(\mathcal{O})}+\|\operatorname{div} w\|_{H^{r-1}(\mathcal{O})}\right.} \\
& \left.+\|\bar{\partial} w \cdot N\|_{H^{r-1.5}(\partial \mathcal{O})}\right]
\end{aligned}
$$

This estimate is well-known and follows from the identity $-\Delta F=\operatorname{curl} \operatorname{curl} F-$ $D \operatorname{div} F$; a convenient reference is Taylor [16]. 
2.3. The curl and divergence estimates of $\eta, v$ and $v_{t}$. Exactly following Section 10 in [6], we have the following

Lemma 2.1. The quantities $D \operatorname{div} \eta^{+}, \operatorname{div} v^{ \pm}, \operatorname{div} v_{t}^{ \pm}$and $D \operatorname{curl} \eta^{+}, \operatorname{curl} v^{ \pm}, \operatorname{curl} v_{t}^{ \pm}$ satisfy the following estimates:

$$
\begin{aligned}
&\left\|\operatorname{div} v_{t}^{ \pm}\right\|_{0.5, \pm}^{2}+\left\|\operatorname{curl} v_{t}^{ \pm}\right\|_{0.5, \pm}^{2}+\left\|\operatorname{div} v^{ \pm}\right\|_{2, \pm}^{2} \\
&+\left\|\operatorname{curl} v^{ \pm}\right\|_{2, \pm}^{2}+\left\|D \operatorname{div} \eta^{+}\right\|_{2.5,+}^{2}+\left\|D \operatorname{curl} \eta^{+}\right\|_{2.5,+}^{2} \\
& \quad \leq C_{\delta} \mathcal{M}_{0}+C_{\delta} T \mathcal{P}\left(\sup _{t \in[0, T]} \mathcal{E}(t)\right)+\delta \sup _{t \in[0, T]} \mathcal{E}(t)
\end{aligned}
$$

where $\delta>0$ is taken sufficiently small, and $P$ denotes a polynomial function of its argument.

2.4. A polynomial-type inequality. For a constant $M \geq 0$, suppose that $f(t) \geq$ $0, t \mapsto f(t)$ is continuous, and

$$
f(t) \leq M+C t P(f(t))
$$

where $P$ denotes a polynomial function, and $C$ is a generic constant. Then for $t$ taken sufficiently small, we have the bound

$$
f(t) \leq 2 M .
$$

This type of inequality, which we introduced in [5], can be viewed as a generalization of standard nonlinear Gronwall inequalities.

2.5. Differentiating the matrix $A$. In this subsection we list a very useful identity here concerning the differentiation of the cofactor matrix $A$ for reference. Let $\delta$ be a differential operator such as $\partial_{t}, \bar{\partial}$ or $D$, then

$$
\delta A_{i}^{j}=-A_{r}^{j} \delta \psi_{, s}^{r} A_{i}^{s} .
$$

For example, when $\delta=\partial_{t}$,

$$
\left(A_{i}^{j}\right)_{t}=-A_{r}^{j} \psi_{t, s}^{r} A_{i}^{s} .
$$

\section{Estimates for Velocity, PREssure, AND their time DeRIVATIVES AT \\ TIME $t=0$}

3.1. Estimates for the initial data. We require estimates for the time derivatives of the velocity and pressure at $t=0$. As in [4], we use $w_{1}, w_{2}, q_{0}$ and $q_{1}$ to denote $v_{t}(0), v_{t t}(0), q(0)$ and $q_{t}(0)$, respectively. Following [4], estimates for $q_{i}$ can be obtained by analyzing certain elliptic equations, and estimates for $w_{i}$ are obtained by letting $t=0$ in (1.3a) and (1.3b). The estimates obtained in [4] are density dependent. In particular, $w_{i}$ and $q_{i}$ satisfy

$$
\left\|w_{1}^{+}\right\|_{2,+}^{2}+\left\|q_{0}^{+}\right\|_{3,+}^{2}+\epsilon\left\|w_{1}^{-}\right\|_{2,-}^{2}+\frac{1}{\epsilon}\left\|q_{0}^{-}\right\|_{3,-}^{2} \leq C \mathcal{P}\left(\left\|u_{0}\right\|_{3, \pm}^{2},|\Gamma|_{4.5}^{2}\right)
$$

and

$$
\left\|w_{2}^{+}\right\|_{0,+}^{2}+\epsilon\left\|w_{2}^{-}\right\|_{0,-}^{2}+\left\|q_{1}^{+}\right\|_{1,+}^{2}+\frac{1}{\epsilon}\left\|q_{1}^{-}\right\|_{1,-}^{2} \leq C \mathcal{P}\left(\left\|u_{0}\right\|_{3, \pm}^{2},|\Gamma|_{4.5}^{2}\right),
$$

where $\mathcal{P}$ is some polynomial of its variables.

However, in order to obtain $\epsilon$-independent estimates, we require $\epsilon$-independent bounds for $q_{i}^{-}$and $w_{i}^{-}$. Indeed, we have the following 
Proposition 3.1. Given $u_{0}^{+} \in H^{3}\left(\Omega^{+}\right), u_{0}^{-} \in H^{3}\left(\Omega^{-}\right)$, and $\Gamma \in H^{4.5}$, then

$$
\begin{aligned}
\left\|w_{1}\right\|_{2, \pm}^{2} & +\left\|w_{2}\right\|_{0, \pm}^{2}+\left\|q_{0}^{+}\right\|_{3,+}^{2}+\left\|q_{1}^{+}\right\|_{1,+}^{2} \\
& +\frac{1}{\epsilon^{2}}\left[\left\|q_{0}^{-}\right\|_{2,-}^{2}+\left\|q_{1}^{-}\right\|_{0,-}^{2}\right] \leq C \mathcal{P}\left(\left\|u_{0}\right\|_{3, \pm}^{2},|\Gamma|_{4.5}^{2}\right) .
\end{aligned}
$$

Proof. We note that the estimates for $w_{i}^{+}$and $q_{i}^{+}$follow from (3.1) and (3.2), so it suffices to obtain estimates for $w_{i}^{-}$and $q_{i}^{-}$. We estimate $q_{0}^{-}$first.

Taking the Lagrangian divergence of (1.3b), by the Lagrangian divergence-free condition $A_{i}^{j} v_{, j}^{-i}=0$, we obtain

$$
\epsilon\left[-A_{i t}^{j} v_{, j}^{-i}-A_{i, \ell}^{j} v_{, j}^{-i} \mathrm{w}^{\ell}+A_{i}^{j} \mathrm{w}_{, j}^{\ell} v_{, \ell}^{-i}\right]+A_{i}^{j}\left(A_{i}^{k} q_{, k}^{-}\right)_{, j}=0 .
$$

Using (2.6) and $\mathrm{w}=A\left(v^{-}-\psi_{t}\right)$ in the equality above at $t=0$, as well as restricting (1.3b) on $\partial \Omega^{-}$in the normal direction at $t=0$, we find that $q_{0}^{-}$satisfies

$$
\begin{array}{ll}
\Delta q_{0}^{-}=-\epsilon\left(D u_{0}^{-}\right)^{T}:\left(D u_{0}^{-}\right) & \text {in } \quad \Omega^{-}, \\
\frac{\partial q_{0}^{-}}{\partial N}=-\epsilon w_{1}^{-} \cdot N-\epsilon\left(\mathrm{w}_{0} \cdot D u_{0}^{-}\right) \cdot N & \text { on } \quad \Gamma, \\
\frac{\partial q_{0}^{-}}{\partial N}=-\epsilon\left(\mathrm{w}_{0} \cdot D u_{0}^{-}\right) \cdot N & \text { on } \quad \partial \mathcal{D} .
\end{array}
$$

By (1.3e),

$$
w_{1}^{-} \cdot N=w_{1}^{+} \cdot N+g_{0}^{\gamma \delta}\left(u_{0, \gamma}^{+} \cdot N\right)\left[\left(u_{0}^{+}-u_{0}^{-}\right) \cdot e_{, \delta}\right] .
$$

Therefore, by elliptic regularity,

$$
\begin{aligned}
\left\|q_{0}^{-}\right\|_{3,-}^{2} \leq C \epsilon^{2}\left[\left\|u_{0, j}^{-i} u_{0, i}^{-j}\right\|_{1,-}^{2}+\left|w_{1}^{+} \cdot N\right|_{1.5}^{2}+\left|g_{0}^{\gamma \delta}\left(u_{0, \gamma}^{+} \cdot N\right)\left(u_{0}^{+}-u_{0}^{-}\right) \cdot e, \delta\right|_{1.5}^{2}\right. \\
\left.\quad+\left|\left(\mathrm{w}_{0} \cdot D u_{0}^{-}\right) \cdot N\right|_{1.5,-}^{2}+\left|\left(\mathrm{w}_{0} \cdot D u_{0}^{-}\right) \cdot N\right|_{1.5, \partial \mathcal{D}}^{2}\right] \\
\leq C \epsilon^{2} \mathcal{P}\left(\left\|u_{0}\right\|_{3, \pm}^{2},|\Gamma|_{4.5}^{2}\right)
\end{aligned}
$$

By (3.6) and (1.3b), we also obtain an $\epsilon$-independent estimate for $w_{1}^{-}$:

$$
\left\|w_{1}^{-}\right\|_{2,-}^{2} \leq C\left[\left\|\mathrm{w}_{0} \cdot D u_{0}^{-}\right\|_{2,-}^{2}+\frac{1}{\epsilon^{2}}\left\|q_{0}^{-}\right\|_{3,-}^{2}\right] \leq C \mathcal{P}\left(\left\|u_{0}\right\|_{3, \pm}^{2},|\Gamma|_{4.5}^{2}\right) .
$$

Similarly, since

$$
w_{2}^{-} \cdot N=w_{2}^{+} \cdot N+2\left(w_{1}^{+}-w_{1}^{-}\right) \cdot n_{t}(0)+\left(u_{0}^{+}-u_{0}^{-}\right) \cdot n_{t t}(0),
$$

by (3.7) we find that

$$
\begin{aligned}
\left|w_{2}^{-} \cdot N\right|_{-0.5}^{2} & \leq C\left[\left|w_{2}^{+} \cdot N\right|_{-0.5}^{2}+\left|w_{1}^{+}-w_{1}^{-}\right|_{1.5}^{2}\left|n_{t}(0)\right|_{0}^{2}+\left|u_{0}^{+}-u_{0}^{-}\right|_{1.5}^{2}\left|n_{t t}(0)\right|_{-0.5}^{2}\right] \\
& \leq C \mathcal{P}\left(\left\|u_{0}\right\|_{3, \pm}^{2},|\Gamma|_{4.5}^{2}\right) .
\end{aligned}
$$

Hence by considering the elliptic problem for $q_{1}^{-}$(see [4] page 14 or letting $t=0$ in (4.11) for the precise equations), the elliptic regularity implies

$$
\left\|q_{1}^{-}\right\|_{1,-}^{2} \leq C \epsilon^{2} \mathcal{P}\left(\left\|u_{0}\right\|_{3, \pm}^{2},|\Gamma|_{4.5}^{2}\right) .
$$

Time-differentiating (1.3b) and setting $t=0$ then yields the estimate

$$
\left\|w_{2}^{-}\right\|_{0,-}^{2} \leq C \mathcal{P}\left(\left\|u_{0}\right\|_{3, \pm}^{2},|\Gamma|_{4.5}^{2}\right) .
$$


Henceforth, we let $\mathcal{M}_{0}$ denote a constant depending on $\left\|u_{0}\right\|_{3, \pm}$ and $|\Gamma|_{4.5}$. Therefore, (3.3) implies

$$
\left\|w_{1}\right\|_{2, \pm}^{2}+\left\|w_{2}\right\|_{0, \pm}^{2}+\left\|q_{0}^{+}\right\|_{3,+}^{2}+\left\|q_{1}^{+}\right\|_{1,+}^{2}+\frac{1}{\epsilon^{2}}\left[\left\|q_{0}^{-}\right\|_{3,-}^{2}+\left\|q_{1}^{-}\right\|_{1,-}^{2}\right] \leq \mathcal{M}_{0} .
$$

In the later discussion, we also need the lower order estimates for $q_{0}^{ \pm}$and $w_{1}^{ \pm}$. Instead of Proposition 3.1, we have the following

Proposition 3.2. Given $u_{0}^{+} \in H^{1.5}\left(\Omega^{+}\right), u_{0}^{-} \in H^{1.5}\left(\Omega^{-}\right)$, and $\Gamma \in H^{3}$, then

$$
\left\|w_{1}\right\|_{0, \pm}^{2}+\left\|q_{0}^{+}\right\|_{1,+}^{2}+\frac{1}{\epsilon^{2}}\left\|q_{0}^{-}\right\|_{1,-}^{2} \leq C \mathcal{P}\left(\left\|u_{0}\right\|_{1.5, \pm}^{2},|\Gamma|_{3}^{2}\right) .
$$

Proof. The parts $\left\|w_{1}^{+}\right\|_{0,+}^{2}$ and $\left\|q_{0}^{+}\right\|_{1,+}^{2}$ in (3.9) follows from [4] by first solving

$$
\begin{aligned}
\Delta q_{0}^{+} & =-u_{0, j}^{+i} u_{0, i}^{+j} & & \text { in } \Omega^{+}, \\
\frac{1}{\epsilon} \Delta q_{0}^{-} & =-u_{0, j}^{-i} u_{0, i}^{-j} & & \text { in } \Omega^{-}, \\
q_{0}^{+}-q_{0}^{-} & =H_{0} & & \text { on } \Gamma, \\
\frac{\partial q_{0}^{+}}{\partial N}-\frac{1}{\epsilon} \frac{\partial q_{0}^{-}}{\partial N} & =\left[\left(u_{0}^{-}-u_{0}^{+}\right) \cdot e_{, \beta} g_{0}^{\alpha \beta} u_{0, \alpha}^{+}+\left(\mathrm{w}_{0} \cdot D u_{0}^{-}\right)\right] \cdot N & & \text { on } \Gamma, \\
\frac{\partial q_{0}^{-}}{\partial N} & =-\epsilon\left(\mathrm{w}_{0} \cdot D u_{0}^{-}\right) \cdot N & & \text { on } \partial \mathcal{D},
\end{aligned}
$$

to obtain that

$$
\begin{aligned}
& \left\|q_{0}^{+}\right\|_{1,+}^{2}+\frac{1}{\epsilon}\left\|q_{0}^{-}\right\|_{1,-}^{2} \leq C\left[\left\|u_{0, j}^{+i} u_{0, i}^{+j}\right\|_{L^{6 / 5}\left(\Omega^{+}\right)}^{4}+\left\|u_{0, j}^{-i} u_{0, i}^{-j}\right\|_{L^{6 / 5}\left(\Omega^{-}\right)}^{4}+\left|H_{0}\right|_{0.5}^{2}\right. \\
& \left.+\left|\left[\left(u_{0}^{-}-u_{0}^{+}\right) \cdot e_{, \beta} g_{0}^{\alpha \beta} u_{0, \alpha}^{+}+\left(\mathrm{w}_{0} \cdot D u_{0}^{-}\right)\right] \cdot N\right|_{-0.5}^{2}+\left|\left(\mathrm{w}_{0} \cdot D u_{0}^{-}\right) \cdot N\right|_{-0.5, \partial \mathcal{D}}^{2}\right] \\
& \quad \leq C \mathcal{P}\left(\left\|u_{0}\right\|_{1.5, \pm}^{2},|\Gamma|_{3}^{2}\right),
\end{aligned}
$$

and the estimate for $w_{1}^{+}$follows from the Euler equations. Then we test (3.4a) against $q_{0}^{-}$to find that

$$
\begin{aligned}
\left\|D q_{0}^{-}\right\|_{0,-}^{2}= & -\int_{\Omega^{-}} \epsilon u_{0, j}^{-i} u_{0, i}^{-j} q_{0}^{-} d x+\int_{\Gamma \cup \partial \mathcal{D}} q_{0}^{-} \frac{\partial q_{0}^{-}}{\partial N} d S \\
\leq & \epsilon\left\|u_{0, j}^{-i} u_{0, i}^{-j}\right\|_{L^{6 / 5}\left(\Omega^{-}\right)}\left\|q_{0}^{-}\right\|_{L^{6}\left(\Omega^{-}\right)}-\epsilon \int_{\Gamma} q_{0}^{-}\left(w_{1}^{-}+\mathrm{w}_{0} \cdot D u_{0}^{-}\right) \cdot N d S \\
& -\epsilon \int_{\partial \mathcal{D}} q_{0}^{-}\left(\mathrm{w}_{0} \cdot D u_{0}^{-}\right) \cdot N d S \\
\leq & C_{\delta} \epsilon^{2} \mathcal{P}\left(\left\|u_{0}\right\|_{1.5, \pm}^{2},|\Gamma|_{3}^{2}\right)+\delta\left\|q_{0}^{-}\right\|_{1,-}^{2},
\end{aligned}
$$

where we use (3.5) to estimate $w_{1}^{-} \cdot N$ in terms of $u_{0}^{ \pm}$and $w_{1}^{+}$. By Poincaré's inequality, we find that (3.9) holds for $\left\|q_{0}^{-}\right\|_{1,-}^{2}$ and therefore, by the Euler equations, for $\left\|w_{1}^{-}\right\|_{0,-}$ as well.

3.2. Basic assumptions on bounds. We assume that we have a sufficiently smooth solution $v^{+}$, such that on the time interval $[0, T]$,

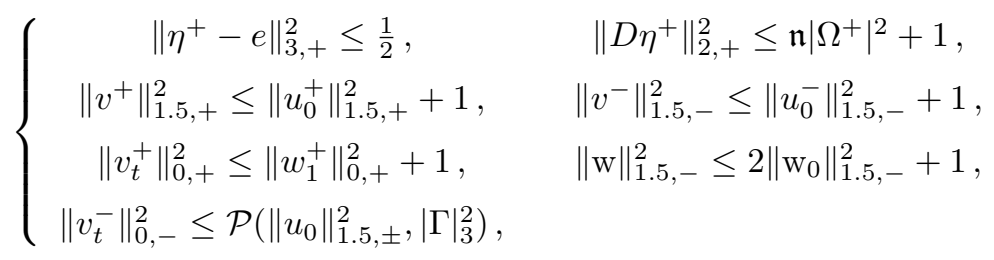


where $\mathcal{P}$ is a polynomial of its variable. Verification of these assumptions, except for $\left\|v_{t}^{-}\right\|_{0,-}$, will follow from the fundamental theorem of calculus, once our energy estimates are completed.

In the following, we allow our generic constant $C$ to depend on the right-hand sides of these inequalities. Given the estimate (3.9), the constant $C$ depends only on the measure of $\Omega^{+},\left\|u_{0}\right\|_{1.5, \pm}^{2}$ and $|\Gamma|_{3}^{2}$.

3.3. The estimates for $\mathbf{w}=A\left(v^{-}-\psi_{t}\right)$ and $\mathbf{w}_{t}$. By the fundamental theorem of Calculus,

$$
\|\mathrm{w}(t)\|_{1.5,-}^{2} \leq 2\left[\|\mathrm{w}(0)\|_{1.5,-}^{2}+t \int_{0}^{t}\left\|\mathrm{w}_{t}(s)\right\|_{1.5,-}^{2} d s\right] .
$$

Since $\mathrm{w}=A\left(v^{-}-\psi_{t}\right)$,

$$
\mathrm{w}_{t}^{j}=-A_{r}^{j} \psi_{t, s}^{r} A_{\ell}^{s}\left(v^{-\ell}-\psi_{t}^{\ell}\right)+A_{\ell}^{j}\left(v_{t}^{-\ell}-\psi_{t t}^{\ell}\right)=-A_{r}^{j} \psi_{t, s}^{r} \mathrm{w}^{s}+A_{\ell}^{j}\left(v_{t}^{-\ell}-\psi_{t t}^{\ell}\right) ;
$$

hence

$$
\left\|\mathrm{w}_{t}\right\|_{0,-}^{2} \leq\|A\|_{L^{\infty}\left(\Omega^{-}\right)}^{2}\left[\left\|D \psi_{t}\right\|_{L^{3}\left(\Omega^{-}\right)}^{2}\|\mathrm{w}\|_{L^{6}\left(\Omega^{-}\right)}^{2}+2\left(\left\|v_{t}^{-}\right\|_{0,-}^{2}+\left\|\psi_{t t}\right\|_{0,-}^{2}\right)\right] \leq C
$$

and

$$
\left\|\mathrm{w}_{t}\right\|_{1.5,-}^{2} \leq C\left[\left\|\psi_{t}\right\|_{2.75,-}^{2}\|\mathrm{w}\|_{1.5,-}^{2}+\left\|v_{t}^{-}\right\|_{1.5,-}^{2}+\left\|\psi_{t t}\right\|_{1.5,-}^{2}\right] \leq C \sup _{t \in[0, T]} \mathcal{E}(t) .
$$

\section{The $\epsilon$-Independent Estimates}

\subsection{Estimates for the pressure and $v_{t t}^{ \pm} \cdot n$.}

Proposition 4.1. Given $\mathcal{E}(t)$ defined in Section 1.5, the solution $v^{ \pm}$of (1.3) satisfies the following estimate:

$$
\left|v_{t t} \cdot n\right|_{-0.5, \pm}^{2}+\left\|q^{+}\right\|_{2.5,+}^{2}+\left\|q_{t}^{+}\right\|_{1,+}^{2}+\frac{1}{\epsilon^{2}}\left[\left\|q^{-}\right\|_{2.5,-}^{2}+\left\|q_{t}^{-}\right\|_{1,-}^{2}\right] \leq C \sup _{t \in[0, T]} \mathcal{E}(t) .
$$

Proof. The proof consists of four steps.

Step 1 (Estimates for $q^{-}$). Since $q^{-}$satisfies

$$
\begin{array}{rlrl}
A_{i}^{j}\left(A_{i}^{k} q_{, k}^{-}\right)_{, j} & =-\epsilon\left[\left(A_{i}^{j}\right)_{t} v_{, j}^{-i}+\left(A_{i}^{j}\right)_{, \ell} \mathrm{w}^{\ell} v_{, j}^{-i}-A_{i}^{j} \mathrm{w}_{, j}^{\ell} v_{, \ell}^{-i}\right] & \text { in } \Omega^{-}, \\
A_{i}^{j} A_{i}^{k} q_{, k}^{-} N_{j} & =\epsilon\left[-v_{t}^{-i}-\mathrm{w}^{\ell} v_{, \ell}^{-i}\right] A_{i}^{j} N_{j} & \text { on } & \Gamma, \\
q_{, k}^{-} N_{k} & =-\epsilon \mathrm{w}^{\ell} v_{, \ell}^{-i} N_{i} & \text { on } \quad \partial \mathcal{D},
\end{array}
$$

elliptic regularity shows that

$$
\begin{aligned}
\frac{1}{\epsilon^{2}}\left\|q^{-}\right\|_{2.5,-}^{2} \leq C & {\left[\left\|A D \mathrm{w} D v^{-}\right\|_{0.5,-}^{2}+\left\|\mathrm{w} D A D v^{-}\right\|_{0.5,-}^{2}+\left\|A_{t} D v^{-}\right\|_{0.5,-}^{2}\right.} \\
& \left.+\left|v_{t}^{-i} n^{i}\right|_{1}+\left|\left(\mathrm{w} \cdot D v^{-}\right) \cdot n\right|_{1}+\left|\left(\mathrm{w} \cdot D v^{-}\right) \cdot N\right|_{1, \partial \mathcal{D}}\right] .
\end{aligned}
$$

We first estimate $\left\|A D \mathrm{w} D v^{-}\right\|_{0.5,-}^{2}$. This requires interpolation, so we first estimate $\left\|A D \mathrm{w} D v^{-}\right\|_{0,-}^{2}$ and $\left\|A D \mathrm{w} D v^{-}\right\|_{1,-}^{2}$. It is easy to see that

$\left\|A D \mathrm{w} D v^{-}\right\|_{0,-}^{2} \leq\|A\|_{L^{\infty}\left(\Omega^{-}\right)}^{2}\|D \mathrm{w}\|_{L^{6}\left(\Omega^{-}\right)}^{2}\left\|D v^{-}\right\|_{L^{3}\left(\Omega^{-}\right)}^{2} \leq C\left[\left\|v^{+}\right\|_{2,+}^{2}+\left\|v^{-}\right\|_{2,-}^{2}\right]$ 
and that

$$
\begin{aligned}
\left\|D\left(A D \mathrm{w} D v^{-}\right)\right\|_{0,-}^{2} & \leq C\left[\left\|D A D \mathrm{w} D v^{-}\right\|_{0,-}^{2}+\left\|A D^{2} \mathrm{w} D v^{-}\right\|_{0,-}^{2}+\left\|A D \mathrm{w} D^{2} v^{-}\right\|_{0,-}^{2}\right] \\
& \leq C\left[\left\|v^{+}\right\|_{3,+}^{2}+\left\|v^{-}\right\|_{3,-}^{2}\right]
\end{aligned}
$$

hence, by interpolation,

$$
\left\|\mathrm{w} D A D v^{-}\right\|_{0.5,-}^{2} \leq C\left[\left\|v^{+}\right\|_{2.5,+}^{2}+\left\|v^{-}\right\|_{2.5,-}^{2}\right] .
$$

Similarly, by interpolation we find that

$$
\left\|\mathrm{w} D A D v^{-}\right\|_{0.5,-}^{2}+\left\|A_{t} D v^{-}\right\|_{0.5,-}^{2} \leq C\left[\left\|v^{+}\right\|_{2.5,+}^{2}+\left\|v^{-}\right\|_{2.5,-}^{2}\right] .
$$

It is also easy to see that $\left|v_{t}^{-} \cdot n\right|_{1} \leq C\left\|v_{t}^{-}\right\|_{1.5}$ and that

$$
\left|\left(\mathrm{w} \cdot D v^{-}\right) \cdot n\right|_{1}^{2}+\left|\left(\mathrm{w} \cdot D v^{-}\right) \cdot N\right|_{1, \partial \mathcal{D}}^{2} \leq C\left\|v^{-}\right\|_{3}^{2} .
$$

It follows that

$$
\frac{1}{\epsilon^{2}}\left\|q^{-}\right\|_{2.5,-}^{2} \leq C \sup _{t \in[0, T]} \mathcal{E}(t) .
$$

Step 2 (Uniform bounds for $v_{t t}^{ \pm} \cdot n$ ). Letting $w=v_{t t}^{+}$in (2.2),

$$
\left|v_{t t}^{+} \cdot n\right|_{-0.5} \leq C\left[\left\|v_{t t}^{+}\right\|_{0,+}+\left\|A_{i}^{j} v_{t t, j}^{+i}\right\|_{H^{1}\left(\Omega^{+}\right)^{\prime}}\right] .
$$

By the incompressibility condition (1.3c),

$$
A_{i}^{j} v_{t t, j}^{+i}=-\left(A_{i}^{j}\right)_{t t} v_{, j}^{+i}-2\left(A_{i}^{j}\right)_{t} v_{t, j}^{+i} .
$$

Let $f \in H^{1}\left(\Omega^{+}\right)$; then

$$
\left\langle\left(A_{i}^{j}\right)_{t t} v_{, j}^{+i}, f\right\rangle_{H^{1}\left(\Omega^{+}\right)}=-\int_{\Omega^{+}}\left(A_{i}^{j}\right)_{t t} v^{+i} f_{, j} d x+\int_{\Gamma}\left(\sqrt{g} n^{i}\right)_{t t} v^{+i} f d S .
$$

Taking the supremum over all $f \in H^{1}\left(\Omega^{+}\right)$with $\|f\|_{1,+}=1$, we find that

$$
\begin{aligned}
& \left\|\left(A_{i}^{j}\right)_{t t} v_{, j}^{+i}\right\|_{H^{1}\left(\Omega^{+}\right)^{\prime}}^{2} \leq C\left[\left\|\left(A_{i}^{j}\right)_{t t} v^{+i}\right\|_{0,+}^{2}+\left|(\sqrt{g} n)_{t t} \cdot v^{+}\right|_{-0.5}^{2}\right] \\
& \quad \leq C\left[\left(\left\|D v_{t}^{+}\right\|_{L^{3}\left(\Omega^{+}\right)}^{2}+\left\|D v^{+}\right\|_{L^{6}\left(\Omega^{+}\right)}^{4}\right)\left\|v^{+}\right\|_{L^{6}\left(\Omega^{+}\right)}^{2}+\left|v_{t}^{+}\right|_{0.5}^{2}+\left|v^{+}\right|_{0.5}^{2}\left|v^{+}\right|_{2.25}^{2}\right] \\
& \quad \leq C\left[\left\|v_{t}^{+}\right\|_{1.5,+}^{2}+\left\|v^{+}\right\|_{3,+}^{2}\right] \leq C \sup _{t \in[0, T]} \mathcal{E}(t) .
\end{aligned}
$$

Similarly,

$$
\left\langle\left(A_{i}^{j}\right)_{t} v_{t, j}^{+i}, f\right\rangle_{H^{1}\left(\Omega^{+}\right)}=-\int_{\Omega^{+}}\left(A_{i}^{j}\right)_{t} v_{t}^{+i} f_{, j} d x+\int_{\Gamma}\left(\sqrt{g} n^{i}\right)_{t} v_{t}^{+i} f d S
$$

which implies

$$
\begin{aligned}
\left\|\left(A_{i}^{j}\right)_{t} v_{t, j}^{+}\right\|_{H^{1}\left(\Omega^{+}\right)^{\prime}}^{2} & \leq C\left[\left\|\left(A_{i}^{j}\right)_{t} v_{t}^{+i}\right\|_{0,+}^{2}+\left|(\sqrt{g} n)_{t} \cdot v_{t}^{+}\right|_{-0.5}^{2}\right] \\
& \leq C\left[\left\|D v^{+}\right\|_{L^{3}\left(\Omega^{+}\right)}^{2}\left\|v_{t}^{+}\right\|_{L^{6}\left(\Omega^{+}\right)}^{2}+\left\|v^{+}\right\|_{W^{1,4}(\Gamma)}^{2}\left\|v_{t}^{+}\right\|_{L^{4}(\Gamma)}^{2}\right] \\
& \leq C\left[\left\|v_{t}^{+}\right\|_{1,+}^{2}+\left\|v^{+}\right\|_{1.5,+}^{4 / 3}\left\|v^{+}\right\|_{3,+}^{2 / 3}\left\|v_{t}^{+}\right\|_{0,+}^{2 / 3}\left\|v_{t}^{+}\right\|_{1.5,+}^{4 / 3}\right] \\
& \leq C\left[\left\|v_{t}^{+}\right\|_{1,+}^{2}+\left\|v^{+}\right\|_{1.5,+}^{2}\left\|v_{t}^{+}\right\|_{1.5,+}^{2}+\left\|v_{t}^{+}\right\|_{0,+}^{2}\left\|v^{+}\right\|_{3,+}^{2}\right] \\
& \leq C \sup _{t \in[0, T]} \mathcal{E}(t) .
\end{aligned}
$$


Therefore,

$$
\left\|A_{i}^{j} v_{t t, j}^{+}\right\|_{H^{1}\left(\Omega^{+}\right)^{\prime}}^{2} \leq C \sup _{t \in[0, T]} \mathcal{E}(t)
$$

and hence

$$
\left|v_{t t}^{+} \cdot n\right|_{-0.5}^{2} \leq C \sup _{t \in[0, T]} \mathcal{E}(t)
$$

The $\epsilon$-independent estimate for $v_{t t}^{-} \cdot n$ is obtained using a different argument. By $(1.3 \mathrm{e})$

$$
v_{t t}^{-} \cdot n=v_{t t}^{+} \cdot n+\left(v^{+}-v^{-}\right) \cdot n_{t t}+2\left(v_{t}^{+}-v_{t}^{-}\right) \cdot n_{t}
$$

hence

$$
\left|v_{t t}^{-} \cdot n\right|_{-0.5}^{2} \leq C\left[\left|v_{t t}^{+} \cdot n\right|_{-0.5}^{2}+\left|\left(v^{+}-v^{-}\right) \cdot n_{t t}\right|_{-0.5}^{2}+\left|\left(v_{t}^{+}-v_{t}^{-}\right) \cdot n_{t}\right|_{-0.5}^{2}\right] .
$$

We claim that for any $f \in H^{1.25}(\Gamma)$ and $g \in H^{-0.5}(\Gamma)$,

$$
|f g|_{-0.5} \leq C|f|_{1.25}|g|_{-0.5} \text {. }
$$

To see this, note that

$$
|f g|_{-0.5}=\sup _{|\phi|_{0.5}=1}\left|\langle f g, \phi\rangle_{H^{0.5}(\Gamma)}\right|=\sup _{|\phi|_{0.5}=1}\left|\langle g, f \phi\rangle_{H^{0.5}(\Gamma)}\right| \leq|g|_{-0.5} \sup _{|\phi|_{0.5}=1}|f \phi|_{0.5} .
$$

It is clear that

$$
|f \phi|_{0} \leq|f|_{L^{\infty}(\Gamma)}|\phi|_{0}
$$

and

$$
|f \phi|_{1}=|f \phi|_{0}+|\bar{\partial} f \phi|_{0}+|f \bar{\partial} \phi|_{0}
$$

By the embeddings

$$
H^{0.25}(\Gamma) \subset L^{8 / 3}(\Gamma), \quad H^{0.75}(\Gamma) \subset L^{8}(\Gamma), \quad H^{1.25}(\Gamma) \subset L^{\infty}(\Gamma),
$$

we see that

$$
|f \phi|_{1} \leq C|f|_{1.25}|\phi|_{1} .
$$

Using interpolation between the inequalities (4.8) and (4.9) shows that

$$
|f \phi|_{0.5} \leq C|f|_{1.25}|\phi|_{0.5},
$$

which in turn proves the claim.

Thus, using the inequality (4.7), we find that

$$
\left|\left(v^{+}-v^{-}\right) \cdot n_{t t}\right|_{-0.5}^{2} \leq C\left|v^{+}-v^{-}\right|_{1.25}^{2}\left[\left|\bar{\partial} v_{t}^{+}\right|_{-0.5}^{2}+\left|v^{+}\right|_{2.25}^{2}\right] \leq C \sup _{t \in[0, T]} \mathcal{E}(t) .
$$

Now we turn to the estimate of the last term $\left|\left(v_{t}^{+}-v_{t}^{-}\right) \cdot n_{t}\right|_{-0.5}^{2}$. By Sobolev's embedding,

$$
\begin{aligned}
\left|v_{t}^{+} \cdot n_{t}\right|_{-0.5}^{2} & \leq C\left|v_{t}^{+} \cdot n_{t}\right|_{0}^{2} \leq C\left\|v_{t}^{+}\right\|_{L^{4}(\Gamma)}^{2}\left\|\bar{\partial} v^{+}\right\|_{L^{4}(\Gamma)}^{2} \leq C\left\|v_{t}^{+}\right\|_{1,+}^{2}\left\|v^{+}\right\|_{2,+}^{2} \\
& \leq C\left[\left\|v_{t}^{+}\right\|_{1.5,+}^{2}\left\|v^{+}\right\|_{1.5,+}^{2}+\left\|v_{t}^{+}\right\|_{0,+}^{2}\left\|v^{+}\right\|_{3,+}^{2}\right] \leq C \sup _{t \in[0, T]} \mathcal{E}(t)
\end{aligned}
$$

The estimate for $v_{t}^{-} \cdot n_{t}$ is the same and thus $v_{t t}^{-} \cdot n$ shares the same $H^{-0.5}(\Gamma)$ bound as $v_{t t}^{+} \cdot n$. Therefore

$$
\left|v_{t t}^{+} \cdot n\right|_{-0.5}^{2}+\left|v_{t t}^{-} \cdot n\right|_{-0.5}^{2} \leq C \sup _{t \in[0, T]} \mathcal{E}(t)
$$


Step 3 (Estimates for $q_{t}^{-}$). Time-differentiating (1.3b) and taking the Lagrangian divergence of the resulting equation, we find that $q_{t}^{-}$satisfies

$$
\begin{aligned}
A_{i}^{j}\left(A_{i}^{k} q_{t, k}^{-}\right)_{, j} & =-\epsilon\left[A_{i}^{j} v_{t t, j}^{-i}+A_{i}^{j}\left(\mathrm{w}^{\ell} v_{, \ell}^{-i}\right)_{t, j}\right]-A_{i}^{j}\left[\left(A_{i}^{k}\right)_{t} q_{, k}^{-}\right]_{, j} & & \text { in } \Omega^{-}, \\
A_{i}^{j} A_{i}^{k} q_{t, k}^{-} N_{j} & =-\left[\epsilon v_{t t}^{-i}+\epsilon\left(\mathrm{w}^{\ell} v_{, \ell}^{-i}\right)_{t}+\left(A_{i}^{k}\right)_{t} q_{, k}^{-}\right] A_{i}^{j} N_{j} & & \text { on } \Gamma, \\
q_{t, k}^{-} N_{k} & =-\epsilon\left(\mathrm{w}^{\ell} v_{, \ell}^{-i}\right)_{t} N_{i} & & \text { on } \partial \mathcal{D} .
\end{aligned}
$$

The goal is to estimate the $H^{1}\left(\Omega^{-}\right)$-norm of $q_{t}^{-}$. By elliptic regularity, it suffices to estimate the $H^{1}(\Omega)^{\prime}$-norm of the interior forcing, and the $H^{-0.5}(\Gamma)$-norm of the boundary forcing.

Similar to Step 2, in order to find an $\epsilon$-independent bound for $\left\|A_{i}^{j} v_{t t, j}^{-}\right\|_{H^{1}\left(\Omega^{-}\right)^{\prime}}^{2}$, we need to estimate $\left\|\left(A_{i}^{j}\right)_{t} v_{t, j}^{-i}\right\|_{H^{1}\left(\Omega^{-}\right)^{\prime}}$. To be more specific, we need to obtain an $\epsilon$-independent bound for $\left|(\sqrt{g} n)_{t} \cdot v_{t}^{-}\right|_{-0.5}$. It suffices to estimate $\left|v_{t}^{-} \cdot n\right|_{-0.5}$ and $\left|v_{t}^{-} \cdot n_{t}\right|_{-0.5}$. It is clear that

$$
\left|v_{t}^{-} \cdot n\right|_{-0.5}^{2} \leq C \sup _{t \in[0, T]} \mathcal{E}(t)
$$

and the estimate for $v_{t}^{-} \cdot n_{t}$ is the same as the one for $v_{t}^{+} \cdot n_{t}$ previously used to establish (4.10); therefore,

$$
\left\|A_{i}^{j} v_{t t, j}^{-i}\right\|_{H^{1}\left(\Omega^{-}\right)^{\prime}}^{2} \leq C \sup _{t \in[0, T]} \mathcal{E}(t) .
$$

We now consider the $H^{1}\left(\Omega^{-}\right)^{\prime}$-norm of $A_{i}^{j}\left(\mathrm{w}^{\ell} v_{, \ell}^{-i}\right)_{t, j}$. Again by (1.3c),

$$
\begin{aligned}
A_{i}^{j}\left(\mathrm{w}^{\ell} v_{, \ell}^{-i}\right)_{t, j}= & A_{i}^{j} \mathrm{w}_{t, j}^{\ell} v_{, \ell}^{-i}+A_{i}^{j} \mathrm{w}_{t}^{\ell} v_{, \ell j}^{-i}+A_{i}^{j} \mathrm{w}_{, j}^{\ell} v_{t, \ell}^{-i} \\
& -\left(A_{i}^{j}\right)_{t} \mathrm{w}^{\ell} v_{, j \ell}^{-i}-\left(A_{i}^{j}\right)_{, \ell} \mathrm{w}^{\ell} v_{t, j}^{-i}-\left(A_{i}^{j}\right)_{t, \ell} \mathrm{w}^{\ell} v_{, j}^{-i} .
\end{aligned}
$$

Similar to (4.4),

$$
\begin{gathered}
\left\langle A_{i}^{j} \mathrm{w}_{t, j}^{\ell} v_{, \ell}^{-i}, f\right\rangle_{H^{1}\left(\Omega^{-}\right)}=-\int_{\Omega^{-}} A_{i}^{j} \mathrm{w}_{t}^{\ell}\left(v_{, \ell}^{-i} f_{, j}+v_{, \ell j}^{-i} f\right) d x+\int_{\Gamma \cup \partial \mathcal{D}} \sqrt{g} n^{i} \mathrm{w}_{t}^{\ell} v_{, \ell}^{-i} f d S \\
=\int_{\Omega^{-}} \mathrm{w}_{t}^{\ell}\left[\left(A_{i}^{j}\right)_{, \ell} v_{, j}^{-i} f-A_{i}^{j} v_{, \ell}^{-i} f_{, j}\right] d x+\int_{\Gamma \cup \partial \mathcal{D}} \sqrt{g} n^{i} \mathrm{w}_{t}^{\ell} v_{, \ell}^{-i} f d S,
\end{gathered}
$$

where $\mathrm{w} \cdot N=0$ on $\Gamma$ and $v^{-} \cdot N=0$ on $\partial \mathcal{D}$ are used in the second equality to eliminate the boundary term due to the integration by parts with respect to $D_{x_{\ell}}$; hence,

$$
\begin{aligned}
\left\|A_{i}^{j} \mathrm{w}_{t, j}^{\ell} v_{, \ell}^{-i}\right\|_{H^{1}\left(\Omega^{-}\right)^{\prime}}^{2} & \leq C\left[\left\|\mathrm{w}_{t} D v^{-}\right\|_{0,+}^{2}+\left\|\mathrm{w}_{t} D A D v^{-}\right\|_{L^{6 / 5}\left(\Omega^{-}\right)}^{2}+\left\|\mathrm{w}_{t} D v^{-}\right\|_{L^{4 / 3}(\Gamma)}^{2}\right] \\
& \leq C \sup _{t \in[0, T]} \mathcal{E}(t) .
\end{aligned}
$$

Similarly, all the other terms in the right-hand side of (4.12) share the same $H^{1}\left(\Omega^{-}\right)^{\prime}$ bound, so that

$$
\left\|A_{i}^{j}\left(\mathrm{w}^{\ell} v_{, \ell}^{-i}\right)_{t, j}\right\|_{H^{1}\left(\Omega^{-}\right)^{\prime}}^{2} \leq C \sup _{t \in[0, T]} \mathcal{E}(t) .
$$

Next, we estimate the $H^{-0.5}(\Gamma)$-norm of the boundary forcing. Because of (4.10), it suffices to estimate $\left|\left(\mathrm{w}^{\ell} v_{, \ell}^{-i}\right)_{t} n^{i}\right|_{-0.5}$. However, because of (4.13), it suffices to estimate $\left\|\left(\mathrm{w}^{\ell} v_{, \ell}^{-}\right)_{t}\right\|_{0,-}$ and it is easy to see that

$$
\left\|\left(\mathrm{w}^{\ell} v_{, \ell}^{-}\right)_{t}\right\|_{0,-}^{2} \leq C\left[\left\|v_{t}^{-}\right\|_{1,-}^{2}+\left\|v_{t}^{+}\right\|_{1,+}^{2}\right] \leq C \sup _{t \in[0, T]} \mathcal{E}(t) .
$$


Combining (4.2) and all the estimates above, we find that for all $t \in[0, T]$,

$$
\frac{1}{\epsilon^{2}}\left\|q_{t}^{-}(t)\right\|_{1,-}^{2} \leq C \sup _{t \in[0, T]} \mathcal{E}(t) .
$$

Step 4 (Estimates for $q^{+}$and $q_{t}^{+}$). By studying the Neumann problems

$$
\begin{array}{ll}
A_{i}^{j}\left(A_{i}^{k} q_{, k}^{+}\right)_{, j}=\epsilon A_{r}^{j} v_{, s}^{+r} A_{j}^{s} v_{, j}^{+i} & \text { in } \Omega^{+}, \\
A_{i}^{j} A_{i}^{k} q_{, k}^{+} N_{j}=-\epsilon v_{t}^{+i} A_{i}^{j} N_{j} & \text { on } \Gamma
\end{array}
$$

and

$$
\begin{array}{rlr}
A_{i}^{j}\left(A_{i}^{k} q_{t, k}^{+}\right)_{, j} & =-\epsilon A_{i}^{j} v_{t t, j}^{+i}+A_{i}^{j}\left[A_{r}^{k} v_{, s}^{+r} A_{i}^{s} q_{, k}^{+}\right]_{, j} & \\
& =\epsilon\left[2 A_{r}^{j} v_{, s}^{+r} A_{i}^{s} v_{t, j}^{+i}+\left(A_{t}^{j}\right)_{t t} v_{, j}^{+i}\right]+A_{i}^{j}\left[A_{r}^{k} v_{, s}^{+r} A_{i}^{s} q_{, k}^{+}\right]_{, j} & \text { in } \Omega^{+}, \\
A_{i}^{j} A_{i}^{k} q_{t, k}^{+} N_{j} & =-\epsilon v_{t t}^{+i} A_{i}^{j} N_{j}-A_{i}^{j} A_{i}^{k} q_{, k}^{+} N_{j} & \text { on } \Gamma,
\end{array}
$$

we obtain that for $t \in[0, T]$,

$$
\left\|q^{+}(t)\right\|_{2.5,+}^{2}+\left\|q_{t}^{+}(t)\right\|_{1,+}^{2} \leq C \sup _{t \in[0, T]} \mathcal{E}(t)
$$

These 4 steps conclude the proposition.

4.2. Uniform boundedness of $\left\|v_{t}^{-}\right\|_{0,-}$. Having $\left\|q_{t}^{-}\right\|_{1,-}^{2}$ uniformly bounded in $\epsilon$, we can prove the last part of the basic assumptions of Section 3.2. By (1.3b), (3.10), (3.9) and (4.1) we find that

$$
\begin{aligned}
\left\|v_{t}^{-}\right\|_{0,-}^{2} & \leq C\left\|\mathrm{w} \cdot D v^{-}\right\|_{0,-}^{2}+\frac{C}{\epsilon^{2}}\left\|q^{-}\right\|_{1,-}^{2} \\
& \leq C\|\mathrm{w}\|_{1,-}^{2}\left\|D v^{-}\right\|_{0.5,-}^{2}+\frac{C}{\epsilon^{2}}\left[\left\|q_{0}^{-}\right\|_{1,-}^{2}+\int_{0}^{t}\left\|q_{t}^{-}\right\|_{1,-}^{2} d s\right] \\
& \leq C+C T \sup _{t \in[0, T]} \mathcal{E}(t) .
\end{aligned}
$$

Therefore, (3.10), (3.9) and (4.1) imply that $\left\|v_{t}^{-}\right\|_{0,-}^{2} \leq C+C T \sup _{t \in[0, T]} \mathcal{E}(t)$ and thus by choosing $T$ small enough, the basic assumptions imply

$$
\|D \psi\|_{2, \pm}^{2}+\|v\|_{1.5, \pm}^{2}+\left\|v_{t}\right\|_{0, \pm}^{2}+\|\mathrm{w}\|_{1.5,-}^{2}+\left\|\mathrm{w}_{t}\right\|_{0,-}^{2} \leq C,
$$

where $C$ is the generic constant defined in Section 3.2.

4.3. Estimates for $v_{t t}^{+} \cdot \bar{\partial} \psi$ and $v_{t t}^{-} \cdot \bar{\partial} \psi$.

Proposition 4.2. Let $v^{ \pm}$be the solution to (1.3). Then

$$
\left|v_{t t}^{+} \cdot \bar{\partial} \psi\right|_{-0.5}^{2}+\left|v_{t t}^{-} \cdot \bar{\partial} \psi\right|_{-0.5}^{2} \leq C \sup _{t \in[0, T]} \mathcal{E}(t)
$$

Proof. First, note that since $D \psi=A^{-1}$, we have

$$
f_{, \gamma}=\psi_{, \gamma}^{i} A_{i}^{j} f_{, j}
$$

It follows from $(1.3 \mathrm{a}),(1.3 \mathrm{~b})$ and $(4.18)$ that

$$
\begin{aligned}
v_{t t}^{+} \cdot \psi_{, \delta} & =-\psi_{, \delta}^{i} A_{i}^{j} q_{t, j}^{+}-\psi_{, \delta}^{i}\left(A_{i}^{j}\right)_{t} q_{, j}^{+}=-q_{t, \delta}^{+}+A_{i}^{j} v_{, \delta}^{+i} q_{, j}^{+}, \\
v_{t t}^{-} \cdot \psi_{, \delta} & =-\frac{1}{\epsilon} q_{t, \delta}^{-}+\frac{1}{\epsilon} A_{i}^{j} v_{, \delta}^{+i} q_{, j}^{-}-\mathrm{w}_{t}^{\ell} v_{, \ell}^{-i} \psi_{, \delta}^{i}-\mathrm{w}^{\ell} v_{t, \ell}^{-i} \psi_{, \delta}^{i} .
\end{aligned}
$$


As a consequence,

$$
\begin{aligned}
\left|v_{t t}^{+} \cdot \psi_{, \delta}\right|_{-0.5}^{2}+\left|v_{t t}^{-} \cdot \psi \psi_{, \delta}\right|_{-0.5}^{2} & \\
\leq & \frac{1}{\epsilon^{2}}\left|q_{t, \delta}^{-}\right|_{-0.5}^{2}+\left|q_{t, \delta}^{+}\right|_{-0.5}^{2}+|A|_{L^{\infty}(\Gamma)}^{2}\left|\bar{\partial} v^{+}\right|_{L^{4}(\Gamma)}^{2}\left[\frac{1}{\epsilon^{2}}\left\|D q^{-}\right\|_{L^{4}(\Gamma)}^{2}+\left\|D q^{+}\right\|_{L^{4}(\Gamma)}^{2}\right] \\
& +\left|\mathrm{w}_{t}\right|_{L^{4}(\Gamma)}^{2}\left|D v^{-}\right|_{L^{4}(\Gamma)}^{2}|\bar{\partial} \psi|_{L^{\infty}(\Gamma)}^{2}+\left|\mathrm{w}^{\ell} v_{t, \ell}^{-} \cdot \bar{\partial} \psi\right|_{-0.5}^{2} \\
\leq & C \sup _{t \in[0, T]} \mathcal{E}(t)+C\left\|v^{+}\right\|_{2,+}^{2}\left[\frac{1}{\epsilon^{2}}\left\|q^{-}\right\|_{2,-}^{2}+\left\|q^{+}\right\|_{2,+}^{2}\right] \\
& +C\left\|\mathrm{w}_{t}\right\|_{1,-}^{2}\left\|v^{-}\right\|_{2,-}^{2}+C\left|\mathrm{w}^{\ell} v_{t, \ell}^{-}\right|_{-0.5}^{2} .
\end{aligned}
$$

By interpolation and Young's inequality,

$$
\begin{aligned}
\left\|v^{+}\right\|_{2,+}^{2}\left[\frac{1}{\epsilon^{2}}\left\|q^{-}\right\|_{2,-}^{2}+\left\|q^{+}\right\|_{2,+}^{2}\right] \leq C\left\|v^{+}\right\|_{1.5,+}^{4 / 3}\left\|v^{+}\right\|_{3,+}^{2 / 3} \times \\
\times\left[\frac{1}{\epsilon^{2}}\left\|q^{-}\right\|_{2.5,-}^{4 / 3}\left\|q^{-}\right\|_{1,-}^{2 / 3}+\left\|q^{+}\right\|_{2.5,+}^{4 / 3}\left\|q^{+}\right\|_{1,+}^{2 / 3}\right] \leq C \sup _{t \in[0, T]} \mathcal{E}(t),
\end{aligned}
$$

and the same upper bound holds for $\left\|\mathrm{w}_{t}\right\|_{1,-}^{2}\left\|v^{-}\right\|_{2,-}^{2}$. For the last term, since $\mathrm{w} \cdot N=0$ on $\Gamma$ and with the identity $\mathrm{w}=(\mathrm{w} \cdot N) N+g_{0}^{\alpha \beta}\left(\mathrm{w} \cdot e_{, \alpha}\right) e_{, \beta}$, we find that

$$
\mathrm{w}^{\ell} v_{t, \ell}^{-}=(\mathrm{w} \cdot N) N^{\ell} v_{t, \ell}^{-}+g_{0}^{\alpha \beta}\left(\mathrm{w} \cdot e_{, \alpha}\right) v_{t, \ell}^{-} e_{, \beta}^{\ell}=g_{0}^{\alpha \beta}\left(\mathrm{w} \cdot e_{, \alpha}\right) v_{t, \beta}^{-} ;
$$

hence

$$
\left|\mathrm{w}^{\ell} v_{t, \ell}^{-}\right|_{-0.5}^{2} \leq C\left|\bar{\partial} v_{t}^{-}\right|_{-0.5}^{2} \leq C \sup _{t \in[0, T]} \mathcal{E}(t) .
$$

(4.17) then follows from summing all the estimates above.

4.4. The $\epsilon$-independent energy estimates. It remains to establish the estimates for $\bar{\partial} v \cdot N, \bar{\partial} v_{t} \cdot N$ and $\bar{\partial} \eta^{+} \cdot N$.

TheOREM 4.1. The solution $v^{ \pm}$to (1.3) satisfies the following estimate:

$$
\begin{aligned}
\sup _{t \in[0, T]} & {\left[\left\|v_{t t}^{+}(t)\right\|_{0,+}^{2}+\epsilon\left\|v_{t t}^{-}(t)\right\|_{0,-}^{2}+\left|\bar{\partial} v_{t}(t) \cdot N\right|_{0, \pm}^{2}+|\bar{\partial} v(t) \cdot N|_{1.5, \pm}^{2}+\left|\bar{\partial} \eta^{+}(t) \cdot N\right|_{3}^{2}\right] } \\
\leq & C_{\delta} \mathcal{M}_{0}+\delta \sup _{t \in[0, T]} \mathcal{E}(t)+C_{\delta} T \mathcal{P}\left(\sup _{t \in[0, T]} \mathcal{E}(t)\right) .
\end{aligned}
$$

Proof. We first derive the estimate

$$
\begin{aligned}
\sup _{t \in[0, T]} & {\left[\left\|v_{t t}^{+}(t)\right\|_{0,+}^{2}+\epsilon\left\|v_{t t}^{-}(t)\right\|_{0,-}^{2}+\left|\bar{\partial} v_{t}(t) \cdot N\right|_{0, \pm}^{2}\right] } \\
\leq & C_{\delta} \mathcal{M}_{0}+\delta \sup _{t \in[0, T]} \mathcal{E}(t)+C_{\delta} T \mathcal{P}\left(\sup _{t \in[0, T]} \mathcal{E}(t)\right) .
\end{aligned}
$$

Twice time-differentiating (1.3a) and (1.3b) and testing the resulting equations against $v_{t t}^{+}$and $v_{t t}^{-}$, respectively, we find that

$$
\begin{aligned}
& \frac{1}{2} \frac{d}{d t}\left[\left\|v_{t t}^{+}\right\|_{0,+}^{2}+\epsilon\left\|v_{t t}^{-}\right\|_{0,-}^{2}\right]+\epsilon \int_{\Omega^{-}}\left[\mathrm{w}_{t t}^{j} v_{, j}^{-i}+2 \mathrm{w}_{t}^{j} v_{t, j}^{-i}+\mathrm{w}^{j} v_{t t, j}^{-i}\right] v_{t t}^{-i} d x \\
& +\int_{\Omega^{+}}\left(A_{i}^{j} q_{, j}^{+}\right)_{t t} v_{t t}^{+i} d x+\int_{\Omega^{-}}\left(A_{i}^{j} q_{, j}^{-}\right)_{t t} v_{t t}^{-i} d x=0 .
\end{aligned}
$$

First, note that $v_{t t, j}^{-i} v_{t t}^{-i}=\frac{1}{2}\left(\left|v_{t t}\right|^{2}\right)_{, j}$, so that

$$
\int_{\Omega^{-}} \mathrm{w}^{j} v_{t t, j}^{-i} v_{t t}^{-i} d x=-\frac{1}{2} \int_{\Omega^{-}} \operatorname{div} \mathrm{w}\left|v_{t t}\right|^{2} d x
$$


Second, using (4.3), we obtain that

$$
\begin{aligned}
\int_{\Omega^{+}}\left(A_{i}^{j} q_{, j}^{+}\right)_{t t} v_{t t}^{+i} d x=-\int_{\Omega^{+}}\left(A_{i}^{j} q^{+}\right)_{t t} v_{t t, j}^{+i} d x+\int_{\Gamma}\left(A_{i}^{j} q^{+}\right)_{t t} v_{t t}^{+i} N_{j} d S \\
=-\int_{\Omega^{+}}\left[\left(A_{i}^{j}\right)_{t t} q^{+}+2\left(A_{i}^{j}\right)_{t} q_{t}^{+}\right] v_{t t, j}^{+i} d x+\int_{\Omega^{+}} q_{t t}^{+}\left[\left(A_{i}^{j}\right)_{t t} v_{, j}^{+i}+2\left(A_{i}^{j}\right)_{t} v_{t, j}^{+i}\right] d x \\
\quad+\int_{\Gamma}\left(A_{i}^{j} q^{+} N_{j}\right)_{t t} v_{t t}^{+i} d S
\end{aligned}
$$

with a similar identity for the term $\int_{\Omega^{-}}\left(A_{i}^{j} q_{, j}^{-}\right)_{t t} v_{t t}^{-i} d x$. Using these identities, (4.21) implies that

$$
\begin{aligned}
& \frac{1}{2} \frac{d}{d t}\left[\left\|v_{t t}^{+}\right\|_{0,+}^{2}+\epsilon\left\|v_{t t}^{-}\right\|_{0,-}^{2}\right]-\frac{\epsilon}{2} \int_{\Omega^{-}} \operatorname{div} \mathrm{w}\left|v_{t t}^{-}\right|^{2} d x+\epsilon \int_{\Omega^{-}}\left[\mathrm{w}_{t t}^{j} v_{, j}^{-i}+\mathrm{w}_{t}^{j} v_{t, j}^{-i}\right] v_{t t}^{-i} d x \\
& -\int_{\Omega^{+}}\left[\left(A_{i}^{j}\right)_{t t} q^{+}+2\left(A_{i}^{j}\right)_{t} q_{t}^{+}\right] v_{t t, j}^{+i} d x-\int_{\Omega^{-}}\left[\left(A_{i}^{j}\right)_{t t} q^{-}+2\left(A_{i}^{j}\right)_{t} q_{t}^{-}\right] v_{t t, j}^{-i} d x \\
& \left.+\int_{\Omega^{+}}\left[\left(A_{i}^{j}\right)_{t t} v_{, j}^{+i}+2\left(A_{i}^{j}\right)_{t} v_{t, j}^{+i}\right] q_{t t}^{+} d x+\int_{\Omega^{-}}\left[\left(A_{i}^{j}\right)_{t t} v_{, j}^{-i}+2\left(A_{i}^{j}\right)_{t} v_{t, j}^{-i}\right] q_{t t}^{-} d x\right\} \\
& +\underbrace{\int_{\Gamma}\left[A_{i}^{j} N_{j}\left(q^{+}-q^{-}\right)\right]_{t t} v_{t t}^{+i} d S}_{\mathrm{O}}+\underbrace{\int_{\Gamma}\left(A_{i}^{j} q^{-} N_{j}\right)_{t t}\left(v_{t t}^{+i}-v_{t t}^{-i}\right) d S}_{\mathrm{J}}=0 .
\end{aligned}
$$

Henceforth, $\mathcal{R}$ denotes lower-order remainder terms that can be easily shown to verify the estimate

$$
\left|\int_{0}^{t} \mathcal{R} d s\right| \leq \mathcal{M}_{0}+C T \mathcal{P}\left(\sup _{t \in[0, T]} \mathcal{E}(t)\right) .
$$

With this notation, the equality above is rewritten as

$$
\frac{1}{2} \frac{d}{d t}\left[\left\|v_{t t}^{+}\right\|_{0,+}^{2}+\epsilon\left\|v_{t t}^{-}\right\|_{0,-}^{2}\right]+\mathrm{O}+\mathrm{I}+\mathrm{J}+\mathcal{R}=0 .
$$

Step 1 (Estimates for the surface tension term $\mathbf{O}$ ). By the boundary condition $(1.3 \mathrm{~d})$,

$$
\int_{\Gamma}\left[A_{i}^{j} N_{j}\left(q^{+}-q^{-}\right)\right]_{t t} v_{t t}^{+i} d S=\int_{\Gamma}\left[\sqrt{g} H n^{j}\right]_{t t} v_{t t}^{+j} d S .
$$

Since the metric $g$ and $H$ are computed from $\eta^{+}$whose time derivatives only involve $\partial_{t}^{k} v^{+}$(which is bounded by $\mathcal{E}(t)$ ), exactly following (12.6) in [6] we find that

$$
\begin{aligned}
& \frac{1}{2} \int_{\Gamma} \sqrt{g} g^{\alpha \beta}\left(v_{t, \alpha}^{+} \cdot n\right)\left(v_{t, \beta}^{+} \cdot n\right) d S \\
& \quad \leq \int_{0}^{t} \int_{\Gamma}\left[A_{i}^{j} N_{j}\left(q^{+}-q^{-}\right)\right]_{t t} v_{t t}^{+i} d S d s+\mathcal{M}_{0}+C T \mathcal{P}\left(\sup _{t \in[0, T]} \mathcal{E}(t)\right) .
\end{aligned}
$$

Step 2 (The estimates for error term I) Let $I=I_{1}+I_{2}+I_{3}+I_{4}$, the summands representing the four integrals contained in I:

$$
\begin{aligned}
& \mathrm{I}_{1}=-\int_{\Omega^{+}}\left[\left(A_{i}^{j}\right)_{t t} q^{+}+2\left(A_{i}^{j}\right)_{t} q_{t}^{+}\right] v_{t t, j}^{+i} d x, \quad \mathrm{I}_{2}=-\int_{\Omega^{-}}\left[\left(A_{i}^{j}\right)_{t t} q^{-}+2\left(A_{i}^{j}\right)_{t} q_{t}^{-}\right] v_{t t, j}^{-i} d x, \\
& \mathrm{I}_{3}=\int_{\Omega^{+}}\left[\left(A_{i}^{j}\right)_{t t} v_{, j}^{+i}+2\left(A_{i}^{j}\right)_{t} v_{t, j}^{+i}\right] q_{t t}^{+} d x, \quad \mathrm{I}_{4}=\int_{\Omega^{-}}\left[\left(A_{i}^{j}\right)_{t t} v_{, j}^{-i}+2\left(A_{i}^{j}\right)_{t} v_{t, j}^{-i}\right] q_{t t}^{-} d x .
\end{aligned}
$$


Integrating by parts in $D_{x_{j}}$ and using the fact that $\left(A_{i}^{j}\right)_{, j}=0$ removing the higher order term $\left[\left(A_{i}^{j}\right)_{t t}\right]_{j}$ which otherwise would have been problematic for our framework, it is easy to see that

$$
\left|\int_{0}^{t}\left(\mathrm{I}_{1}+\mathrm{I}_{2}\right) d s\right| \leq C T \mathcal{P}\left(\sup _{t \in[0, T]} \mathcal{E}(t)\right)
$$

Next, we estimate $\mathrm{I}_{4}$; the estimate for $\mathrm{I}_{3}$ will follow in the same fashion. Integrating by parts in time, the most problematic terms to estimate are denoted by

$$
\mathrm{I}_{41}=\int_{0}^{t} \int_{\Omega^{-}} A_{r}^{j} \psi_{t t t, s}^{r} A_{i}^{s} v_{, j}^{-i} q_{t}^{-} d x d s \quad \text { and } \quad \mathrm{I}_{42}=\int_{0}^{t} \int_{\Omega^{-}}\left(A_{i}^{j}\right)_{t} v_{t t, j}^{-i} q_{t}^{-} d x d s
$$

The same as the estimates for $\mathrm{I}_{1}$ and $\mathrm{I}_{2}$, integrating by parts with respect to $D_{x_{s}}$ for $\mathrm{I}_{41}$ and $D_{x_{j}}$ for $\mathrm{I}_{42}$, we find that

$$
\left|\int_{0}^{t}\left(\mathrm{I}_{41}+\mathrm{I}_{42}\right) d s\right| \leq C T \mathcal{P}\left(\sup _{t \in[0, T]} \mathcal{E}(t)\right)
$$

so that

$$
\left|\int_{0}^{t} \mathrm{I} d s\right| \leq \mathcal{M}_{0}+C T \mathcal{P}\left(\sup _{t \in[0, T]} \mathcal{E}(t)\right)
$$

where $\mathcal{M}_{0}$ comes from the temporal boundary terms appearing when integrating by parts in time for $\mathrm{I}_{3}$ and $\mathrm{I}_{4}$.

Step 3 (The estimates for the error term J) Since $A^{T} N=\sqrt{g} n$, we find that

$$
\begin{aligned}
\mathrm{J}= & \underbrace{\int_{\Gamma}\left(\sqrt{g} q^{-}\right)_{t t}\left(v_{t t}^{+}-v_{t t}^{-}\right) \cdot n d S}_{\mathrm{J}_{1}}+2 \underbrace{\int_{\Gamma}\left(\sqrt{g} q^{-}\right)_{t} n_{t}^{i} \cdot\left(v_{t t}^{+i}-v_{t t}^{-i}\right) d S}_{\mathrm{J}_{2}} \\
& +\underbrace{\int_{\Gamma} \sqrt{g} q^{-}\left(v_{t t}^{+}-v_{t t}^{-}\right) \cdot n_{t t} d S}_{\mathrm{J}_{3}}
\end{aligned}
$$

The estimates for $\mathbf{J}_{1}$ : by the boundary condition (1.3e),

$$
\begin{aligned}
\left(v_{t t}^{+}-v_{t t}^{-}\right) \cdot n= & \left(v^{-}-v^{+}\right) \cdot n_{t t}+2\left(v_{t}^{-}-v_{t}^{+}\right) \cdot n_{t} \\
= & {\left[\left(v^{+}-v^{-}\right) \cdot \psi_{, \delta}\right] g^{\gamma \delta}\left(v_{t, \gamma}^{+} \cdot n\right)+2\left[\left(v_{t}^{+}-v_{t}^{-}\right) \cdot \psi_{, \delta}\right] g^{\gamma \delta}\left(v_{, \gamma}^{+} \cdot n\right) } \\
& +\mathcal{P}_{i}\left(\bar{\partial} \psi, \bar{\partial} v^{+}\right)\left(v^{+i}-v^{-i}\right) .
\end{aligned}
$$


Time integrating $\mathrm{J}_{1}$ and integrating by parts in time, we find that

$$
\begin{aligned}
\int_{0}^{t} \mathrm{~J}_{1} d s= & \underbrace{\left[\int_{\Gamma}\left(\sqrt{g} q^{-}\right)_{t}\left(v_{t t}^{+}-v_{t t}^{-}\right) \cdot n d S\right](t)}_{\mathrm{J}_{10}}-\int_{\Gamma}\left(\sqrt{g} q^{-}\right)_{t}(0)\left(w_{2}^{+}-w_{2}^{-}\right) \cdot N d S \\
& -\int_{0}^{t} \int_{\Gamma}\left(\sqrt{g} q^{-}\right)_{t} g^{\gamma \delta}\left[\left(v^{+}-v^{-}\right) \cdot \psi_{, \delta}\right]\left(v_{t t, \gamma}^{+} \cdot n\right) d S d s \quad\left(\equiv \mathrm{J}_{11}\right) \\
& -3 \int_{0}^{t} \int_{\Gamma}\left(\sqrt{g} q^{-}\right)_{t} g^{\gamma \delta}\left[\left(v_{t}^{+}-v_{t}^{-}\right) \cdot \psi_{, \delta}\right]\left(v_{t, \gamma}^{+} \cdot n\right) d S d s \\
& -2 \int_{0}^{t} \int_{\Gamma}\left(\sqrt{g} q^{-}\right)_{t} g^{\gamma \delta}\left[\left(v_{t t}^{+}-v_{t t}^{-}\right) \cdot \psi_{, \delta}\right]\left(v_{, \gamma}^{+} \cdot n\right) d S d s \quad\left(\equiv \mathrm{J}_{12}\right) \\
& +\int_{0}^{t} \int_{\Gamma}\left(\sqrt{g} q^{-}\right)_{t}\left[\mathcal{P}_{i j}\left(\bar{\partial} \psi, \bar{\partial} v^{+}\right) \bar{\partial} v_{t}^{+i}+\mathcal{P}_{j}\left(\bar{\partial} \psi, \bar{\partial} v^{+}\right)\right]\left(v^{+j}-v^{-j}\right) d S d s \\
= & \mathrm{J}_{10}+\mathrm{J}_{11}+\mathrm{J}_{12}-\int_{\Gamma}\left(\sqrt{g} q^{-}\right)_{t}(0)\left(w_{2}^{+}-w_{2}^{-}\right) \cdot N d S+\int_{0}^{t} \mathcal{R} d s .
\end{aligned}
$$

By (4.17),

$$
\left|\mathrm{J}_{12}\right| \leq C T \mathcal{P}\left(\sup _{t \in[0, T]} \mathcal{E}(t)\right),
$$

so we only need to estimate $\mathrm{J}_{10}$ and $\mathrm{J}_{11}$.

For the temporal boundary term $\mathrm{J}_{10}$, using (4.23), the fundamental theorem of calculus, and interpolation, we find that

$$
\begin{aligned}
\left|\mathrm{J}_{10}\right| & \leq C\left\|v_{t}^{+}\right\|_{1,+}\left\|q_{t}^{-}\right\|_{1,-}+C T \mathcal{P}\left(\sup _{t \in[0, T]} \mathcal{E}(t)\right) \\
& \leq C_{\delta}\left\|v_{t}^{+}\right\|_{0}^{2}+\delta\left[\left\|v_{t}^{+}\right\|_{1.5}^{2}+\left\|q_{t}^{-}\right\|_{1}^{2}\right]+C T \mathcal{P}\left(\sup _{t \in[0, T]} \mathcal{E}(t)\right) \\
& \leq C_{\delta}+C T \mathcal{P}\left(\sup _{t \in[0, T]} \mathcal{E}(t)\right)+\delta \sup _{t \in[0, T]} \mathcal{E}(t)
\end{aligned}
$$

As for $\mathrm{J}_{11}$, integrating by parts with respect to $\bar{\partial}_{\gamma}$,

$$
\left.\begin{array}{rl}
\mathrm{J}_{11}= & \int_{0}^{t} \int_{\Gamma}\left(\sqrt{g} q^{-}\right)_{t, \gamma} g^{\gamma \delta}\left[\left(v^{+}-v^{-}\right) \cdot \psi_{, \delta}\right]\left(v_{t t}^{+} \cdot n\right) d S d s \quad\left(\equiv \mathrm{J}_{111}\right) \\
& +\int_{0}^{t} \int_{\Gamma}\left(\sqrt{g} q^{-}\right)_{t}\left[\mathcal{P}_{i}\left(\bar{\partial}^{2} \psi\right)\left(v^{+i}-v^{-i}\right)+\mathcal{P}(\bar{\partial} \psi, \bar{\partial} v)\right]\left(v_{t t}^{+} \cdot n\right) d S d s \\
& +\int_{0}^{t} \int_{\Gamma}\left(\sqrt{g} q^{-}\right)_{t} \mathcal{P}_{i j}^{\alpha}(\bar{\partial} \psi) \bar{\partial}^{2} \psi^{i}\left(v^{+j}-v^{-j}\right)\left(v_{t t}^{+} \cdot \psi_{, \alpha}\right) d S d s
\end{array}\right\} \equiv \mathrm{J}_{112} .
$$

By $H^{0.5}(\Gamma)-H^{-0.5}(\Gamma)$ duality, we find that $\mathrm{J}_{112}$ is bounded by $C T \mathcal{P}\left(\sup _{t \in[0, T]} \mathcal{E}(t)\right)$.

For the term $\mathrm{J}_{111}$, we add and subtract $q^{+}$to obtain

$$
\begin{aligned}
\mathrm{J}_{111}= & \int_{0}^{t} \int_{\Gamma}\left[\sqrt{g}\left(q^{-}-q^{+}\right)\right]_{t, \gamma} g^{\gamma \delta}\left[\left(v^{+}-v^{-}\right) \cdot \psi_{, \delta}\right]\left(v_{t t}^{+} \cdot n\right) d S d s \\
& +\int_{0}^{t} \int_{\Gamma}\left(\sqrt{g} q^{+}\right)_{t, \gamma} g^{\gamma \delta}\left[\left(v^{+}-v^{-}\right) \cdot \psi_{, \delta}\right]\left(v_{t t}^{+} \cdot n\right) d S d s \\
= & \int_{0}^{t} \int_{\Gamma}\left[\left(\sqrt{g} g^{\alpha \beta} \psi_{, \alpha}\right)_{, \beta} \cdot n\right]_{t, \gamma} g^{\gamma \delta}\left[\left(v^{+}-v^{-}\right) \cdot \psi_{, \delta}\right]\left(v_{t t}^{+} \cdot n\right) d S d s \quad\left(\equiv \mathrm{J}_{1111}\right) \\
& +\int_{0}^{t} \int_{\Gamma}\left(\sqrt{g} q^{+}\right)_{t, \gamma} g^{\gamma \delta}\left[\left(v^{+}-v^{-}\right) \cdot \psi_{, \delta}\right]\left(v_{t t}^{+} \cdot n\right) d S d s \quad\left(\equiv \mathrm{J}_{1112}\right) .
\end{aligned}
$$


In order to study the term $\mathrm{J}_{1111}$, we integrate by parts with respect to $\bar{\partial}_{\gamma}$ and then in time (to move one time derivative from $v_{t t}^{+} \cdot n$ ) and find that the most challenging term to estimate is

$$
\int_{0}^{t} \int_{\Gamma}\left[\left(\sqrt{g} g^{\alpha \beta} \psi, \alpha\right)_{, \beta} \cdot n\right]_{t t} g^{\gamma \delta}\left[\left(v^{+}-v^{-}\right) \cdot \psi_{, \delta}\right]\left(v_{t}^{+} \cdot n\right)_{, \gamma} d S d s
$$

Since

$$
\begin{aligned}
& {\left[\left(\sqrt{g} g^{\alpha \beta} \psi_{, \alpha}\right)_{, \beta} \cdot n\right]_{t t}=\left(\sqrt{g} g^{\alpha \beta} v_{t, \alpha}^{+}\right)_{, \beta} \cdot n+\sqrt{g} g^{\alpha \beta} g^{\gamma \delta}\left(v_{t, \gamma}^{+} \cdot \psi_{, \delta}\right)\left(\psi_{, \alpha \beta} \cdot n\right)} \\
& -\sqrt{g}\left[g^{\alpha \gamma} g^{\beta \delta}\left(v_{t, \gamma}^{+} \cdot \psi_{, \delta}+v_{t, \delta}^{+} \cdot \psi_{, \gamma}\right)+g^{\alpha \beta} g^{\gamma \delta}\left(v_{, \gamma}^{+} \cdot n\right)\left(v_{, \delta}^{+} \cdot n\right)\right](\psi, \alpha \beta \cdot n) \\
& \quad+\mathcal{P}_{i}^{1}\left(\bar{\partial} \psi, \bar{\partial} v^{+}\right) \bar{\partial}^{2} \psi^{i}+\mathcal{P}_{i}^{2}\left(\bar{\partial} \psi, \bar{\partial} v^{+}\right) \bar{\partial}^{2} v^{+i}
\end{aligned}
$$

the most difficult term to estimate after integrating by parts with respect to $\bar{\partial}_{\beta}$ is

$$
\int_{0}^{t} \int_{\Gamma} \sqrt{g} g^{\alpha \beta} g^{\gamma \delta}\left[\left(v^{+}-v^{-}\right) \cdot \psi_{, \delta}\right]\left(v_{t, \alpha}^{+} \cdot n\right)\left(v_{t, \beta}^{+} \cdot n\right){ }_{, \gamma} d S d s .
$$

Now by

$$
g^{\alpha \beta}\left(v_{t, \alpha}^{+} \cdot n\right)\left(v_{t, \beta}^{+} \cdot n\right)_{, \gamma}=\frac{1}{2}\left[g^{\alpha \beta}\left(v_{t, \alpha}^{+} \cdot n\right)\left(v_{t, \beta}^{+} \cdot n\right)\right]_{, \gamma}-\frac{1}{2}\left(g^{\alpha \beta}\right)_{, \gamma}\left(v_{t, \alpha}^{+} \cdot n\right)\left(v_{t, \beta}^{+} \cdot n\right),
$$

integrating by parts implies that the above integral is bounded by $C T \mathcal{P}\left(\sup _{t \in[0, T]} \mathcal{E}(t)\right)$. Therefore,

$$
\left|\mathrm{J}_{1111}\right| \leq \mathcal{M}_{0}+C T \mathcal{P}\left(\sup _{t \in[0, T]} \mathcal{E}(t)\right)+|\underbrace{\int_{\Gamma}(\sqrt{g} H)_{t} g^{\gamma \delta}\left[\left(v^{+}-v^{-}\right) \cdot \psi_{, \delta}\right]\left(v_{t}^{+} \cdot n\right)_{, \gamma} d S}_{\mathrm{K}_{1}}|,
$$

where $\mathcal{M}_{0}$ and the term $\mathrm{K}_{1}$ arises from the temporal boundary term when integrating by parts in time for $\mathrm{J}_{1111}$. However, similar to the estimate of $\mathrm{J}_{10}$, the temporal boundary term $\mathrm{K}_{1}$ can be estimated as

$$
\begin{aligned}
\left|\mathrm{K}_{1}\right| & \leq C\left\|v^{+}\right\|_{2.5,+}\left\|v_{t}^{+}\right\|_{1.5,+}+C T \mathcal{P}\left(\sup _{t \in[0, T]} \mathcal{E}(t)\right) \\
& \leq C_{\delta}\left\|v^{+}\right\|_{0,+}^{2}+\delta\left[\left\|v^{+}\right\|_{3,+}^{2}+\left\|v_{t}^{+}\right\|_{1.5,+}^{2}\right]+C T \mathcal{P}\left(\sup _{t \in[0, T]} \mathcal{E}(t)\right) \\
& \leq C_{\delta}+\delta \sup _{t \in[0, T]} \mathcal{E}(t)+C T \mathcal{P}\left(\sup _{t \in[0, T]} \mathcal{E}(t)\right) .
\end{aligned}
$$

As for $\mathrm{J}_{1112}$, by the divergence theorem, since $A_{i}^{j} v_{t t, j}^{+i}$ is a lower order term thanks to incompressibility, we find that

$$
\begin{aligned}
\mathrm{J}_{1112}= & \underbrace{\int_{0}^{t} \int_{\mathcal{U}} \chi \frac{1}{\sqrt{g}}\left(\sqrt{g} q^{+}\right)_{t, \gamma j} g^{\gamma \delta}\left[\left(v^{+}-v^{-}\right) \cdot \psi_{, \delta}\right] A_{i}^{j} v_{t t}^{+i} d x d s}_{\mathrm{K}_{2}} \\
& +\int_{0}^{t} \int_{\mathcal{U}} \chi \frac{1}{\sqrt{g}}\left(\sqrt{g} q^{+}\right)_{t, \gamma} g^{\gamma \delta}\left[\left(v^{+}-v^{-}\right) \cdot \psi_{, \delta}\right] A_{i}^{j} v_{t t, j}^{+i} d x d s \\
& +\int_{0}^{t} \int_{\mathcal{U}}\left(\sqrt{g} q^{+}\right)_{t, \gamma}\left[\chi \frac{1}{\sqrt{g}} g^{\gamma \delta}\left[\left(v^{+}-v^{-}\right) \cdot \psi_{, \delta}\right]\right]_{, j} A_{i}^{j} v_{t t}^{+i} d x d s=\mathrm{K}_{2}+\int_{0}^{t} \mathcal{R} d s,
\end{aligned}
$$

where $\chi$ is a smooth cut-off function supported around $\Gamma$ with $\chi=1$ on $\Gamma$, and with the support of $\chi$ taken sufficiently close to $\Gamma$ so that the tangential derivatives are well-defined. We set

$$
\mathcal{U}=\operatorname{supp}(\chi) \cap \Omega^{+} .
$$


To estimate $\mathrm{K}_{2}$, the structure of the Euler equations has to be used. In fact,

$$
|\mathrm{K}_{2}+\underbrace{\int_{0}^{t} \int_{\mathcal{U}} \chi v_{t t, \gamma}^{+i} g^{\gamma \delta}\left[\left(v^{+}-v^{-}\right) \cdot \psi, \delta\right] v_{t t}^{+i} d x d s}_{\mathrm{K}_{3}}| \leq C T \mathcal{P}\left(\sup _{t \in[0, T]} \mathcal{E}(t)\right) .
$$

where we use the identity

$$
-\left(\sqrt{g} v_{t}^{+i}\right)_{t, \gamma}=\left(\sqrt{g} A_{i}^{j} q_{, j}^{+}\right)_{t, \gamma}=A_{i}^{j}\left(\sqrt{g} q_{, j}^{+}\right)_{t, \gamma}-\left(A_{i}^{j}\right)_{t}\left(\sqrt{g} q_{, j}^{+}\right)_{, \gamma}-\left(A_{i}^{j}\right)_{, \gamma}\left(\sqrt{g} q_{, j}^{+}\right)_{t}
$$

to replace $\frac{1}{\sqrt{g}} A_{i}^{j}\left(\sqrt{g} q^{+}\right)_{t, \gamma j}$ in $\mathrm{K}_{2}$ by $v_{t t, \gamma}^{+i}$ in $\mathrm{K}_{3}$.

It then suffices to estimate $\mathrm{K}_{3}$ to complete the estimate of $\mathrm{J}_{1}$. However, since $2 v_{t t, \gamma}^{+i} v_{t t}^{+i}=\left(\left|v_{t t}^{+}\right|^{2}\right)_{, \gamma}$, integrating by parts with respect to $\bar{\partial}_{\gamma}$ implies that $\mathrm{K}_{3}$ is bounded by $C T \mathcal{P}\left(\sup _{t \in[0, T]} \mathcal{E}(t)\right)$.

Therefore, combining all the estimates above we find that

$$
\left|\int_{0}^{t} \mathrm{~J}_{1} d s\right| \leq\left(C_{\delta}+\mathcal{M}_{0}\right)+C T \mathcal{P}\left(\sup _{t \in[0, T]} \mathcal{E}(t)\right)+\delta \sup _{t \in[0, T]} \mathcal{E}(t)
$$

The estimates for $\mathbf{J}_{2}$ : we first note that by (4.19),

$$
\left(v_{t t}^{+}-v_{t t}^{-}\right) \cdot \psi_{, \delta}=\frac{1}{\epsilon} q_{t, \delta}^{-}-q_{t, \delta}^{+}+A_{i}^{j} v_{, \delta}^{+i}\left(\frac{1}{\epsilon} q_{, j}^{+}-q_{, j}^{-}\right)+\mathrm{w}_{t}^{\ell} v_{, \ell}^{-i} \psi_{, \delta}^{i}+\mathrm{w}^{\ell} v_{t, \ell}^{-i} \psi_{, \delta}^{i}
$$

Therefore, by $n_{t}=-g^{\gamma \delta}\left(v_{, \gamma}^{+} \cdot n\right) \psi_{, \delta}$ and (4.25), we find that

$$
\begin{aligned}
\mathrm{J}_{2}= & -\int_{\Gamma}\left(\sqrt{g} q^{-}\right)_{t} g^{\gamma \delta}\left(v_{, \gamma}^{+} \cdot n\right)\left[\frac{1}{\epsilon} q_{t, \delta}^{-}-q_{t, \delta}^{+}+A_{i}^{j} v_{, \delta}^{+i}\left(\frac{1}{\epsilon} q_{, j}^{+}-q_{, j}^{-}\right)\right] d S \\
& -\int_{\Gamma}\left(\sqrt{g} q^{-}\right)_{t} g^{\gamma \delta}\left(v_{, \gamma}^{+} \cdot n\right)\left[\mathrm{w}_{t}^{\ell} v_{, \ell}^{-i} \psi_{, \delta}^{i}+\mathrm{w}^{\ell} v_{t, \ell}^{-i} \psi_{, \delta}^{i}\right] d S .
\end{aligned}
$$

It is easy to see that the second integral is bounded by $C \epsilon \mathcal{P}\left(\sup _{t \in[0, T]} \mathcal{E}(t)\right)$ (the presence of $\epsilon$ is due to the estimate of $\left.\left(\sqrt{g} q^{-}\right)_{t}\right)$. The most problematic term of the first integral is when the time derivative acts on $q$ and in this case, by $H^{0.5}(\Gamma)-H^{-0.5}(\Gamma)$ duality, (4.2), (4.14) and (4.15) imply that

$$
\left|\int_{\Gamma}\left(\sqrt{g} q^{-}\right)_{t} g^{\gamma \delta}\left(v_{, \gamma}^{+} \cdot n\right)\left[\frac{1}{\epsilon} q_{t, \delta}^{-}-q_{t, \delta}^{+}+A_{i}^{j} v_{, \delta}^{+i}\left(\frac{1}{\epsilon} q_{, j}^{+}-q_{, j}^{-}\right)\right] d S\right| \leq C \epsilon \mathcal{P}\left(\sup _{t \in[0, T]} \mathcal{E}(t)\right) .
$$

Therefore,

$$
\int_{0}^{t} \mathrm{~J}_{2} d s \leq C \epsilon T \mathcal{P}\left(\sup _{t \in[0, T]} \mathcal{E}(t)\right)
$$

The estimates for $\mathbf{J}_{3}$ : by $(4.25)$ we find that

$$
\begin{aligned}
\mathrm{J}_{3}= & -\int_{\Gamma} q^{-} \sqrt{g} g^{\gamma \delta}\left(v_{t, \gamma}^{+} \cdot n\right)\left[\left(v_{t t}^{+}-v_{t t}^{-}\right) \cdot \psi, \delta\right] d S+\int_{\Gamma} \mathcal{P}_{i}\left(\bar{\partial} \psi, \bar{\partial} v^{+}\right) q^{-}\left(v_{t t}^{+i}-v_{t t}^{-i}\right) d S \\
= & -\underbrace{\int_{\Gamma} \sqrt{g} g^{\gamma \delta} q^{-}\left(v_{t, \gamma}^{+} \cdot n\right)\left(\frac{1}{\epsilon} q_{t, \delta}^{-}-q_{t, \delta}^{+}\right) d S}_{\mathrm{K}_{4}}-\int_{\Gamma} \sqrt{g} g^{\gamma \delta} q^{-}\left(v_{t, \gamma}^{+} \cdot n\right) A_{i}^{j} v_{, \delta}^{+i}\left(\frac{1}{\epsilon} q_{t, j}^{+}-q_{t, j}^{-}\right) d S \\
& -\int_{\Gamma} \sqrt{g} g^{\gamma \delta} q^{-}\left(v_{t, \gamma}^{+} \cdot n\right)\left[\mathrm{w}_{t}^{\ell} v_{, \ell}^{-i} \psi_{, \delta}^{i}+\mathrm{w}^{\ell} v_{t, \ell}^{-i} \psi_{, \delta}^{i}\right] d S+\int_{\Gamma} \mathcal{P}_{i}\left(\bar{\partial} \psi, \bar{\partial} v^{+}\right) q^{-}\left(v_{t t}^{+i}-v_{t t}^{-i}\right) d S \\
= & -\mathrm{K}_{4}+\mathcal{R} .
\end{aligned}
$$


Before estimating $\mathrm{K}_{4}$, we first note that by the divergence theorem,

$$
\begin{aligned}
\int_{\Gamma} & \sqrt{g} g^{\gamma \delta} q^{-}\left(v_{t, \gamma}^{+} \cdot n\right) q_{t, \delta}^{+} d S=\int_{\Gamma} q^{-} g^{\gamma \delta}\left(v_{t, \gamma}^{+i} A_{i}^{j} N_{j}\right) q_{t, \delta}^{+} d S \\
= & \int_{\mathcal{U}} \chi q^{-} g^{\gamma \delta} A_{i}^{j} v_{t, \gamma}^{+i} q_{t, \delta j}^{+} d x+\int_{\mathcal{U}} \chi q^{-} g^{\gamma \delta} A_{i}^{j} v_{t, \gamma j}^{+i} q_{t, \delta}^{+} d x+\int_{\mathcal{U}}\left(\chi q^{-} g^{\gamma \delta}\right)_{, j} A_{i}^{j} v_{t, \gamma}^{+i} q_{t, \delta}^{+} d x
\end{aligned}
$$

We then follow the estimate of $\mathrm{J}_{1112}$ and obtain the inequality

$$
\left|\int_{\mathcal{U}} \chi q^{-} g^{\gamma \delta} A_{i}^{j} v_{t, \gamma}^{+i} q_{t, \delta j}^{+} d x+\int_{\mathcal{U}} \chi q^{-} g^{\gamma \delta} v_{t, \gamma}^{+i} v_{t t, \delta}^{+i} d x\right| \leq C \epsilon \mathcal{P}\left(\sup _{t \in[0, T]} \mathcal{E}(t)\right)
$$

By interpolating $v_{t}^{+} \in H^{1}\left(\Omega^{+}\right)$between $L^{2}\left(\Omega^{+}\right)$and $H^{1.5}\left(\Omega^{+}\right)$,

$$
\begin{aligned}
\left|\int_{\mathcal{U}} \chi q^{-} g^{\gamma \delta} v_{t, \gamma}^{+i} v_{t, \delta}^{+i} d x\right| & \leq C\left\|q^{-}\right\|_{L^{6}\left(\Omega^{-}\right)}\left\|v_{t}^{+}\right\|_{W^{1,3}\left(\Omega^{+}\right)}\left\|v_{t}^{+}\right\|_{1,+} \\
& \leq C_{\delta} \epsilon\left[\mathcal{M}_{0}+T \sup _{t \in[0, T]} \mathcal{E}(t)\right]\left\|v_{t}^{+}\right\|_{1,+}^{2}+\epsilon \delta\left\|v_{t}^{+}\right\|_{1.5,+}^{2} \\
& \leq C_{\delta} \epsilon\left[\mathcal{M}_{0}+T \sup _{t \in[0, T]} \mathcal{E}(t)\right]+\epsilon \delta \sup _{t \in[0, T]} \mathcal{E}(t)
\end{aligned}
$$

Therefore,

$$
\begin{aligned}
\left|\int_{0}^{t} \int_{\Gamma} \sqrt{g} g^{\gamma \delta} q^{-}\left(v_{t, \gamma}^{+} \cdot n\right) q_{t, \delta}^{+} d S d s\right| \\
\leq \epsilon \mathcal{M}_{0}+C \epsilon T \mathcal{P}\left(\sup _{t \in[0, T]} \mathcal{E}(t)\right)+\left|\left[\int_{\mathcal{U}} \chi q^{-} g^{\gamma \delta} v_{t, \gamma}^{+i} v_{t, \delta}^{+i} d x\right](t)\right| \\
\leq C_{\delta} \in \mathcal{M}_{0}+C_{\delta} \epsilon T \mathcal{P}\left(\sup _{t \in[0, T]} \mathcal{E}(t)\right)+\epsilon \delta \sup _{t \in[0, T]} \mathcal{E}(t)
\end{aligned}
$$

It remains to estimate $\frac{1}{\varepsilon} \int_{\Gamma} \sqrt{g} g^{\gamma \delta} q^{-}\left(v_{t, \gamma}^{+} \cdot n\right) q_{t, \delta}^{-} d S$. By adding and subtracting $q^{+}$, using (1.3d) and (4.28) we find that

$$
\begin{aligned}
\frac{1}{\epsilon} \mid \int_{0}^{t} \int_{\Gamma} & \sqrt{g} g^{\gamma \delta} q^{-}\left(v_{t, \gamma}^{+} \cdot n\right) q_{t, \delta}^{-} d S d s \mid \\
= & \frac{1}{\epsilon}\left|\int_{0}^{t} \int_{\Gamma} \sqrt{g} g^{\gamma \delta} q^{-}\left(v_{t, \gamma}^{+} \cdot n\right)\left(q^{-}-q^{+}+q^{+}\right)_{t, \delta} d S d s\right| \\
\leq & C_{\delta} \mathcal{M}_{0}+C_{\delta} T \mathcal{P}\left(\sup _{t \in[0, T]} \mathcal{E}(t)\right)+\delta \sup _{t \in[0, T]} \mathcal{E}(t) \\
& +\frac{1}{\epsilon}\left|\int_{0}^{t} \int_{\Gamma} \sqrt{g} g^{\gamma \delta} q^{-}\left(v_{t, \gamma}^{+} \cdot n\right)\left[\frac{1}{\sqrt{g}}\left(\sqrt{g} g^{\alpha \beta} \psi_{, \alpha}\right)_{, \beta} \cdot n\right]_{t, \delta} d S d s\right|
\end{aligned}
$$

As for the last term, since $\psi_{, \alpha} \cdot n=0$, the most problematic term appears when the time derivative hits $\psi, \alpha$. Integrating by parts with respect to $\bar{\partial}_{\beta}$ for that term, 
we obtain that

$$
\begin{aligned}
& \int_{\Gamma} \sqrt{g} g^{\gamma \delta} q^{-}\left(v_{t, \gamma}^{+} \cdot n\right)\left[\frac{1}{\sqrt{g}}\left(\sqrt{g} g^{\alpha \beta} \psi_{, \alpha}\right)_{, \beta} \cdot n\right]_{t, \delta} d S \\
&=-\int_{\Gamma} \sqrt{g} g^{\alpha \beta} g^{\gamma \delta} q^{-}\left(v_{t, \beta \gamma}^{+} \cdot n\right)\left(v_{, \alpha \delta}^{+} \cdot n\right) d S+\int_{\Gamma} \bar{\partial} q^{-}\left(\bar{\partial} v_{t}^{+} \cdot n\right) \mathcal{P}(\bar{\partial} \psi) \bar{\partial}^{2} v^{+} d S \\
&+\int_{\Gamma} q^{-}\left(\bar{\partial} v_{t}^{+} \cdot n\right) \mathcal{P}_{i}\left(\bar{\partial} \psi, \bar{\partial} v^{+}\right) \bar{\partial}^{3} \psi^{i} d S \\
&+\int_{\Gamma} q^{-}\left(\bar{\partial} v_{t}^{+} \cdot n\right)\left[\mathcal{P}_{i j}^{1}\left(\bar{\partial} \psi, \bar{\partial} v^{+}\right) \bar{\partial}^{2} \psi^{j}+\mathcal{P}_{i j}^{2}\left(\bar{\partial} \psi, \bar{\partial} v^{+}\right) \bar{\partial}^{2} v^{+j}\right] \bar{\partial}^{2} \psi^{i} d S \\
&=-\frac{1}{2} \frac{\partial}{\partial t} \int_{\Gamma} \sqrt{g} g^{\alpha \beta} g^{\gamma \delta} q^{-} v_{, \beta \gamma}^{+} v_{, \alpha \delta}^{+}+\mathcal{R} .
\end{aligned}
$$

Consequently,

$$
\begin{aligned}
\frac{1}{\epsilon}\left|\int_{0}^{t} \int_{\Gamma} \sqrt{g} g^{\gamma \delta} q^{-}\left(v_{t, \gamma}^{+} \cdot n\right)\left[\frac{1}{\sqrt{g}}\left(\sqrt{g} g^{\alpha \beta} \psi_{, \alpha}\right)_{, \beta} \cdot n\right]_{t, \delta} d S d s\right| \\
\leq C_{\delta} \mathcal{M}_{0}+C_{\delta} T \mathcal{P}\left(\sup _{t \in[0, T]} \mathcal{E}(t)\right)+\delta \sup _{t \in[0, T]} \mathcal{E}(t) \\
+\frac{C}{\epsilon}\left|\left[\int_{\Gamma} \sqrt{g} g^{\gamma \delta} q^{-}\left(v_{, \alpha \delta}^{+} \cdot n\right)\left(v_{, \beta \gamma}^{+} \cdot n\right) d S\right](t)\right| .
\end{aligned}
$$

Similar to (4.27), we find that

$$
\begin{aligned}
\frac{1}{\epsilon}\left|\left[\int_{\Gamma} \sqrt{g} g^{\gamma \delta} q^{-}\left(v_{, \alpha \delta}^{+} \cdot n\right)\left(v_{, \beta \gamma}^{+} \cdot n\right) d S\right](t)\right| \\
\quad \leq C_{\delta}\left[\mathcal{M}_{0}+T \mathcal{P}\left(\sup _{t \in[0, T]} \mathcal{E}(t)\right)\right]+\delta \sup _{t \in[0, T]} \mathcal{E}(t)
\end{aligned}
$$

The estimate of $\mathrm{J}_{3}$ then follows from combining the above estimates:

$$
\left|\int_{0}^{t} \mathrm{~J}_{3} d s\right| \leq C_{\delta} \mathcal{M}_{0}+C_{\delta} T \mathcal{P}\left(\sup _{t \in[0, T]} \mathcal{E}(t)\right)+\delta \sup _{t \in[0, T]} \mathcal{E}(t) .
$$

Combining (4.22), (4.24), (4.26) and (4.29), we find that

$$
\begin{aligned}
\sup _{t \in[0, T]}\left[\left\|v_{t t}^{+}(t)\right\|_{0,+}^{2}+\epsilon\left\|v_{t t}^{-}(t)\right\|_{0,-}^{2}+\left|\bar{\partial} v_{t}^{+}(t) \cdot n(t)\right|_{0}^{2}\right] \\
\leq C_{\delta} \mathcal{M}_{0}+\delta \sup _{t \in[0, T]} \mathcal{E}(t)+C_{\delta} T \mathcal{P}\left(\sup _{t \in[0, T]} \mathcal{E}(t)\right) .
\end{aligned}
$$

It follows that

$$
\begin{aligned}
\sup _{t \in[0, T]}\left[\left\|v_{t t}^{+}(t)\right\|_{0,+}^{2}+\epsilon\left\|v_{t t}^{-}(t)\right\|_{0,-}^{2}+\left|\bar{\partial} v_{t}(t) \cdot N\right|_{0, \pm}^{2}\right] \\
\leq C_{\delta} \mathcal{M}_{0}+\delta \sup _{t \in[0, T]} \mathcal{E}(t)+C_{\delta} T \mathcal{P}\left(\sup _{t \in[0, T]} \mathcal{E}(t)\right)
\end{aligned}
$$

by the jump condition $v^{+} \cdot n=v^{-} \cdot n$ and the fact that $n(t)=N+\int_{0}^{t} n_{t} d s$.

Step 4 (Estimates for $|\bar{\partial} v \cdot N|_{1.5, \pm}$ and $\left|\bar{\partial} \eta^{+} \cdot N\right|_{3}$ ).

Our goal is to establish an inequality of the type

$$
\sup _{t \in[0, T]} \mathcal{E}(t) \leq C_{\delta} M_{0}+\delta \sup _{t \in[0, T]} \mathcal{E}(t)+C_{\delta} \mathcal{P}\left(\sup _{t \in[0, T]} \mathcal{E}(t)\right)
$$


with $\epsilon$-independent $C_{\delta}$. By (2.4), we only need (4.30), (4.31) and (4.32) to ensure the $\epsilon$-independence of $C_{\delta}$. The real difficult part is estimate (4.30) which we prove in details. Following the proof of (12.33) and (12.34) in [6], by defining

$$
\mathcal{E}_{k}(t)=|\bar{\partial} \psi \cdot N|_{3}^{2}+\sum_{\ell=0}^{k}\left\|\partial_{t}^{\ell} v\right\|_{3-1.5 \ell, \pm}^{2}
$$

the proof in $[6]$ implies

$$
\begin{aligned}
|\bar{\partial} v \cdot N|_{1.5, \pm}^{2} & \leq C_{\delta} \mathcal{M}_{0}+\delta \mathcal{E}_{1}(t)+C_{\delta} \mathcal{P}\left(\sup _{t \in[0, T]} \mathcal{E}_{1}(t)\right), \\
|\bar{\partial} \psi \cdot N|_{3}^{2} & \leq C_{\delta} \mathcal{M}_{0}+\delta \mathcal{E}_{0}(t)+C_{\delta} \mathcal{P}\left(\sup _{t \in[0, T]} \mathcal{E}_{0}(t)\right) .
\end{aligned}
$$

Since $\mathcal{E}_{k}(t) \leq \mathcal{E}(t)$, we obtain that

$$
\sup _{t \in[0, T]}|\bar{\partial} v(t) \cdot N|_{1.5, \pm}^{2} \leq C_{\delta} \mathcal{M}_{0}+\delta \sup _{t \in[0, T]} \mathcal{E}(t)+C_{\delta} T \mathcal{P}\left(\sup _{t \in[0, T]} \mathcal{E}(t)\right)
$$

and

$$
\sup _{t \in[0, T]}|\bar{\partial} \psi(t) \cdot N|_{3}^{2} \leq C_{\delta} \mathcal{M}_{0}+\delta \sup _{t \in[0, T]} \mathcal{E}(t)+C_{\delta} T \mathcal{P}\left(\sup _{t \in[0, T]} \mathcal{E}(t)\right) .
$$

Estimates (4.30)-(4.32) then conclude Theorem 4.1.

4.5. A uniform bound for $\mathcal{E}(t)$. Using (2.3), combining estimates (2.4) and (4.30)-(4.32), we find that for all $t \in[0, T]$,

$$
\mathcal{E}(t) \leq \mathcal{M}_{0}+C T \mathcal{P}\left(\sup _{t \in[0, T]} \mathcal{E}(t)\right)
$$

This is the polynomial inequality (2.5) that we had sought. It follows that by taking $T>0$ sufficiently small,

$$
\sup _{t \in[0, T]} \mathcal{E}(t) \leq 2 \mathcal{M}_{0}
$$

Finally, choose $T>0$ even smaller so that the fundamental theorem of calculus ensures that the basic assumptions of Section 3.2 are satisfied.

\section{The Limit AS $\epsilon \rightarrow 0$}

Having established our $\epsilon$-independent estimate (4.33), we can now pass to the limit as $\epsilon \rightarrow 0$, and show that we recover the solutions of the one-phase Euler equations (1.4).

Let $\varphi \in \mathcal{C}^{\infty}\left(\mathbb{R}^{\mathfrak{n}}\right)$ so that $\varphi \cdot N=0$ on $\partial \mathcal{D}$. Testing (1.3) against $\varphi$, since $\varphi$ is continuous across $\Gamma$, we find that

$$
\begin{array}{r}
\int_{\Omega^{+}} v_{t}^{+i} \varphi^{i} d x-\int_{\Omega^{+}} q^{+} A_{i}^{k} \varphi_{, k}^{i} d x+\int_{\Gamma}\left(q^{+}-q^{-}\right) A_{i}^{k} N_{k} \varphi^{i} d S \\
+\epsilon \int_{\Omega^{-}} v_{t}^{-i} \varphi^{i} d x+\epsilon \int_{\Omega^{-}} \mathrm{w}^{j} v_{, j}^{-i} \varphi^{i} d x-\int_{\Omega^{-}} q^{-} A_{i}^{k} \varphi_{, k}^{i} d x=0 .
\end{array}
$$


Note that $v^{+}, v^{-}, q^{+}$and $q^{-}$above depend on $\epsilon$ implicitly. Our a priori bound (4.33) allows us to find sequences, still parameterized by $\epsilon$, such that $\epsilon \rightarrow 0$,

$$
\begin{array}{lll}
v_{t}^{+} \rightarrow \mathfrak{v}_{t} & \text { in } & L^{2}\left(0, T ; H^{1.5}\left(\Omega^{+}\right)\right), \\
v^{+} \rightarrow \mathfrak{v} & \text { in } & L^{2}\left(0, T ; H^{2}\left(\Omega^{+}\right)\right), \\
v^{+} \rightarrow \mathfrak{v} & \text { in } & L^{2}\left(0, T ; H^{3}\left(\Omega^{+}\right)\right), \\
q^{+} \rightarrow \mathfrak{q} & \text { in } & L^{2}\left(0, T ; H^{2.5}\left(\Omega^{+}\right)\right) .
\end{array}
$$

Let $\tilde{\psi}=e+\int_{0}^{t} \mathfrak{v} d s$. By $(5.2 \mathrm{~b}), \psi \rightarrow \tilde{\psi}$ in $L^{\infty}\left(0, T ; H^{2}\left(\Omega^{+}\right)\right)$; hence $A \rightarrow \mathcal{A}:=$ $(D \tilde{\psi})^{-1}$ in $L^{\infty}\left(0, T ; H^{1}\left(\Omega^{+}\right)\right)$and $\Delta_{g} \psi \rightarrow \Delta_{\tilde{g}} \tilde{\psi}$ in $L^{\infty}\left(0, T ; H^{0.5}(\Gamma)\right)$ with $\tilde{g}_{\alpha \beta}=$ $\tilde{\psi}_{, \alpha} \cdot \tilde{\psi}_{, \beta}$ because of $(5.2 \mathrm{c})$. Therefore, by (1.3d), (5.1) converges to

$$
\int_{\Omega^{+}} \mathfrak{v}_{t}^{i} \varphi^{i} d x-\int_{\Omega^{+}} \mathfrak{q} \mathcal{A}_{i}^{k} \varphi_{, k}^{i} d x+\int_{\Gamma} \mathcal{H} \mathcal{A}_{i}^{k} N_{k} \varphi^{i} d S=0,
$$

where $\mathcal{H}=-\Delta_{\tilde{g}} \tilde{\psi} \cdot \tilde{n}$. This shows that $U=\mathfrak{v} \circ \tilde{\psi}^{-1}$ and $P=\mathfrak{q} \circ \tilde{\psi}^{-1}$ solve (1.4).

Acknowledgments. SS was supported by the National Science Foundation under grant DMS-0701056.

\section{REFERENCES}

[1] D.M. Ambrose, Well-posedness of vortex sheets with surface tension, SiAm J. Math. Anal. 35 (2003), no. 1, 211-244.

[2] D.M. Ambrose and N. Masmoudi, The zero surface tension limit of two-Dimensional Water waves, Comm. Pure Appl. Math., 58 (2005), 1287-1315.

[3] D.M. Ambrose and N. Masmoudi, Well-posedness of 3D vortex sheets with surface TEnsion, Comm. Math. Sci., 5(2), (2007), 391-430.

[4] C.H. A. Cheng, D. Coutand and S. Shkoller, On the motion of vortex sheets with Surface tension in the 3D Euler equations with vorticity, Comm. Pure Appl. Math., 61(12), (2008), 1715-1752.

[5] D. Coutand and S. Shkoller, On the interaction Between QUaSilinear elastodynamics and the Navier-Stokes equations, Arch. Rational Mech. Anal. 179(3), (2006), 303-352.

[6] D. Coutand and S. Shkoller, Well-Posedness of the FreE-Surface INCOMPREssible Euler Equations with or without surface tension, J. Amer. Math. Soc., 20, (2007), 829-930.

[7] B. Dacorogna and J. Moser, On a PARTial Differential EQUATION InVolving the JACOBian Determinant, Annales de l'institut Henri Poincare' (C) Analyse non line'aire, 7, (1990), $1-26$.

[8] W. Craig, An existence theory for water waves and the Boussinesq And Korteweg-De Vries scaling limits, Comm. Partial Differential Equations, 10 (1985), no. 8, 787-1003.

[9] V. Kamotski and G. Lebeau, On 2D Rayleigh-Taylor instabilities, Asymptot. Anal. 42 (2005), 1-27.

[10] D. Lannes, Well-posedness of the water-waves equations, J. Amer. Math. Soc., 18, (2005) 605-654

[11] H. Lindblad, Well-Posedness for the motion of AN inCOMPRESSible Liquid With Free SURFACE BOUNDARY, Annals of Math., 162, (2005), 109-194.

[12] V.I. Nalimov, The Cauchy-Poisson Problem (in Russian), Dynamika Splosh. Sredy, 18 (1974),104-210.

[13] B. Schweizer, On the three-dimensional Euler EQuations with a Free Boundary subJeCt to surface tension, Ann. Inst. H. Poincaré Anal. Non Linéaire, 22 (2005), 753-781.

[14] J. Shatah, C. Zeng, Geometry and a Priori estimates for free boundary problems of The Euler's equation, Comm. Pure Appl. Math., 61(5), (2008), 698-744.

[15] J. Shatah, C. Zeng, A priori estimates for fluid interface Problems, Comm. Pure Appl. Math. 61, (2008), 848-876.

[16] M. Taylor, Partial Differential Equations Volume I Basic Theory, Springer Verlag

[17] R. Temam, Navier-Stokes Equations: Theory and Numerical Analysis, Amer. Math. Soc., 2001. 
[18] S. Wu, Well-posedness in Sobolev spaces of the full Water Wave Problem in 2-D, Invent. Math., 130 (1997), 39-72.

[19] S. Wu, Well-posedness in Sobolev spaces of the full Water wave Problem in 3-D, J. Amer. Math. Soc., 12 (1999), 445-495.

[20] S. Wu, Mathematical analysis of vortex sheets, Comm. Pure Appl. Math., 59, (2006), 1065-1206.

[21] H. Yosihara, Gravity Waves on the Free Surface of an Incompressible Perfect Fluid, Publ. RIMS Kyoto Univ., 18 (1982), 49-96.

[22] H. Yoshihra, Capillary-gravity waves for an incompressible ideal fluid, J. Math. Kyoto Univ. 23 (1983), no. 4, 649-694.

E-mail address: cchsiao@math.ncu.edu.tw

Center for Scientific Computation And Mathematical Modeling, University of Maryland, College Park, MD 20742

Department of Mathematics, National Central University, Jhongli City, Taoyuan County 32001, Taiwan (R.O.C.)

E-mail address: D.Coutand@ma.hw.ac.uk

CANPDE, Maxwell Institute for Mathematical Sciences and department of Mathematics, Heriot-Watt University, Edinburgh, EH14 4AS, UK

E-mail address: shkoller@math.ucdavis.edu

Department of Mathematics, University of California, Davis, CA 95616 